\title{
Lateralized Expression of Cortical Perineuronal Nets during Maternal Experience is Dependent on MECP2
}

\author{
(DBilly Y. B. Lau, Dana E. Layo, Bett Emery, ${ }^{-}$Matthew Everett, Anushree Kumar, \\ (-) Parker Stevenson, ${ }^{-K}$ Kristopher G. Reynolds, Andrew Cherosky, Sarah-Anne H. Bowyer, Sarah Roth, \\ Delaney G. Fisher, ${ }^{\circledR}$ Rachel P. McCord, and $\mathbb{D}^{-K e e r t h i}$ Krishnan
}

https://doi.org/10.1523/ENEURO.0500-19.2020

Department of Biochemistry and Cellular and Molecular Biology, University of Tennessee, Knoxville, TN 37996

\begin{abstract}
Cortical neuronal circuits along the sensorimotor pathways are shaped by experience during critical periods of heightened plasticity in early postnatal development. After closure of critical periods, measured histologically by the formation and maintenance of extracellular matrix structures called perineuronal nets (PNNs), the adult mouse brain exhibits restricted plasticity and maturity. Mature PNNs are typically considered to be stable structures that restrict synaptic plasticity on cortical parvalbumin + $(\mathrm{PV}+)$ GABAergic neurons. Changes in environment (i.e., novel behavioral training) or social contexts (i.e., motherhood) are known to elicit synaptic plasticity in relevant neural circuitry. However, little is known about concomitant changes in the PNNs surrounding the cortical PV+ GABAergic neurons. Here, we show novel changes in PNN density in the primary somatosensory cortex (SS1) of adult female mice after maternal experience [called surrogate (Sur)], using systematic microscopy analysis of a whole brain region. On average, PNNs were increased in the right barrel field and decreased in the left forelimb regions. Individual mice had left hemisphere dominance in PNN density. Using adult female mice deficient in methyl-CpGbinding protein 2 (MECP2), an epigenetic regulator involved in regulating experience-dependent plasticity, we found that MECP2 is critical for this precise and dynamic expression of PNN. Adult naive Mecp2-heterozygous (Het) females had increased PNN density in specific subregions in both hemispheres before maternal experience, compared with wild-type (WT) littermate controls. The laterality in PNN expression seen in naive Het (NH) was lost after maternal experience in Sur Het (SH) mice, suggesting possible intact mechanisms for plasticity. Together, our results identify subregion and hemisphere-specific alterations in PNN expression in adult females, suggesting extracellular matrix plasticity as a possible neurobiological mechanism for adult behaviors in rodents.
\end{abstract}

Key words: alloparenting; lateralization; MECP2; perineuronal nets; Rett syndrome; somatosensory cortex

\section{Significance Statement}

Perineuronal nets (PNNs) are extracellular matrix structures that surround cortical parvalbumin $+(P V+)$ fast spiking GABAergic interneurons and synapses. They have long been considered stable structures that restrict synaptic plasticity. Removal of PNNs by enzymes reactivates plasticity in the rodent visual and auditory cortices and in the amygdala. However, it is currently unknown whether PNNs in adult brains undergo changes in expression under normal physiological conditions, similar to synaptic plasticity mechanisms. If they do, PNNs may not be very stable structures as they are perceived. We provide evidence that mature PNNs in the adult mouse primary somatosensory cortex (SS1) show dynamic expression changes in a hemisphere-specific, subregion-specific manner after maternal experience and are regulated by methyl-CpGbinding protein 2 (MECP2). 


\section{Introduction}

Perineuronal nets (PNNs) are specialized extracellular matrix structures that can act as physical barriers or modulators of plasticity, restrict axon regeneration, and form molecular brakes that actively control synaptic maturation and the function of cortical parvalbumin+ $(\mathrm{PV}+)$ GABAergic interneurons that drive $\gamma$ oscillations (Nakagawa et al., 1986; Kosaka and Heizmann, 1989; Hartig et al., 1992; Bartos et al., 2002; Deepa et al., 2002; Pizzorusso et al., 2002, 2006; Dityatev et al., 2007; Frischknecht et al., 2009; Sugiyama et al., 2009; Gundelfinger et al., 2010; Durand et al., 2012; Orlando et al., 2012; Suttkus et al., 2012; Donato et al., 2013; Vo et al., 2013; Ye and Miao, 2013; Krishnan et al., 2015, 2017; Bernard and Prochiantz, 2016; Carstens et al., 2016; de Winter et al., 2016; Begum and Sng, 2017; Hou et al., 2017; Kalemaki et al., 2018; Ueno et al., 2018; Sigal et al., 2019). In rodents, mature PNNs in the adult cortex are thought to be stable structures, inhibitory to plasticity, and perhaps play roles in long-term memory such as "engrams" (Gogolla et al., 2009; Carstens et al., 2016; Thompson et al., 2018). However, most of these observations are based on postnatal cortical development (when typical connections in neural circuitry are still forming) and models for neurobiological disorders (where neural circuitry development and function have gone awry). Currently, it is unclear whether changes in adult PNN expression occur under normal conditions and behavioral contexts.

PNNs are composed of chondroitin sulfate proteoglycans, hyaluronan glycosaminoglycan chains, link proteins and tenascin-R (Bignami et al., 1992; Carulli et al., 2007; Kwok et al., 2010; Miyata and Kitagawa, 2017). Wisteria floribunda agglutinin (WFA) is commonly used as a marker for PNNs in the cortex and other brain regions (Hartig et al., 1992; Brückner et al., 1996). WFA specifically binds to $\mathrm{N}$-acetyl galactosamine found on most chondroitin sulfate

Received December 2, 2019; accepted April 14, 2020; First published April 22, 2020.

The authors declare no competing financial interests.

Author contributions: B.Y.B.L. and K.K. designed research; B.Y.B.L., D.E.L., B.E., M.E., A.K., P.S., K.G.R., A.C., S.-A.H.B., S.R., D.G.F., and K.K. performed research; B.Y.B.L., R.P.M., D.G.F., and K.K. analyzed data; B.Y.B.L., D.E.L., and K.K. wrote the paper.

This work was supported by a postdoctoral fellowship award from Rettsyndrome.org (B.Y.B.L.), research assistantships from the University of Tennessee-Knoxville (D.G.F., B.E., M.E., P.S.), and startup funds from the University of Tennessee-Knoxville (K.K.).

Acknowledgements: K.K. thanks her mentors Dr. Weifang Shen and the late Dr. Corey Largman at the Veterans Affairs Medical Center, University of California, San Francisco, for fostering in her the joy for science and the scientific process, during the gap years after her undergraduate education. We also thank the following students in helping us get started with this project and for technical contributions: Ashlee Tannehill, Taryn Lester, Ronald Dean Franz, Marty Lay, and Simran Dayal.

Correspondence should be addressed to Keerthi Krishnan at krishnan@ utk.edu.

https://doi.org/10.1523/ENEURO.0500-19.2020

Copyright @ 2020 Lau et al.

This is an open-access article distributed under the terms of the Creative Commons Attribution 4.0 International license, which permits unrestricted use, distribution and reproduction in any medium provided that the original work is properly attributed. side chains of chondroitin sulfate proteoglycans. In rodents, WFA-labeled PNNs are localized predominantly around soma and proximal dendrites of PV+ GABAergic interneurons of the mature cortex. They interdigitate with synaptic contacts on cortical PV+ GABAergic neurons and regulate experience-dependent synaptic plasticity in the cortex, hippocampus, and amygdala (Gogolla et al., 2009; Miyata et al., 2012; Krishnan et al., 2015, 2017; Cattaud et al., 2018; Murthy et al., 2019; Sigal et al., 2019).

In the human brain, decreased numbers of PNNs are associated with pathologic conditions such as decreased memory and motor agility (Morawski et al., 2004; Brückner et al., 2008; Cabungcal et al., 2013; Pantazopoulos et al., 2015; Enwright et al., 2016). Mouse models for varying neurologic disorders show abnormal/ atypical expression of PNNs which, when removed, can greatly improve the associated pathology or behavioral readouts in these models (Berretta et al., 2015; Pizzo et al., 2016; Krishnan et al., 2017; Reinhard et al., 2019). We have previously shown that precocious or atypical expression of PNNs caused sensory processing deficits in developing male or adult female mouse models for Rett syndrome, respectively (Krishnan et al., 2015, 2017). Rett syndrome is a neuropsychiatric disorder predominantly caused by mutations in the X-linked gene, methyl-CpG-binding protein 2 (MECP2; Amir et al., 1999). MECP2 regulates neuronal chromatin architecture and gene transcription in response to neural activity and experience during postnatal life (Zhou et al., 2006; Chahrour et al., 2008; Skene et al., 2010; Ebert et al., 2013; Becker et al., 2016). The known cellular function of MECP2 and the characteristic timing of disease progression led us to hypothesize that MECP2 regulates experience-dependent plasticity in specific neural circuits during windows of enhanced sensory and social experience throughout life; disruptions in timing of these plasticity mechanisms results in atypical responses in behavior. We previously tested this hypothesis using a pup retrieval task in the alloparental care paradigm (Krishnan et al., 2017).

Parenting is an ethologically relevant social behavior consisting of stereotypic components involving the care and nourishment of young. First-time dams seek and gather wandering/scattered pups back to the nest (pup retrieval), an essential aspect of maternal care. Pup retrieval involves processing of primary sensory cues (auditory, tactile, olfactory) to direct efficient searching and gathering of pups with goal-directed movements back to the nest (Beach and Jaynes, 1956; Stern, 1996; Lonstein et al., 2015). Virgin female mice (naive) with no previous maternal experience can execute efficient pup retrieval after co-housing [surrogates (Sur)] with a first-time mother and her pups (Cohen et al., 2011a). This assay allows for interrogation of adult experience-dependent plasticity mechanisms as well as likely non-hormonal, epigenetic mechanisms involving the sensory and motor neural circuits (Stolzenberg and Champagne, 2016). The role of the auditory cortex in pup retrieval and maternal experience is a topic of investigation in many labs (Stiebler et al., 1997; 
Galindo-Leon et al., 2009; Cohen et al., 2011a; Marlin et al., 2015). They continue to contribute to the understanding of how relevant sensory cues are processed in the maternal brain.

Previously, we found that atypical increase in PNN expression in the auditory cortex of Mecp2-heterozygous (Het) females caused inefficient pup retrieval (Krishnan et al., 2017). However, we did not find discernable changes in PNN density in the wild-type (WT) auditory cortex after successful completion of the pup retrieval task. On the one hand, as PNNs are considered barriers to plasticity, we anticipated reduction in PNN expression in WT that could facilitate efficient retrieval. On the other hand, there are no known reports of reduction in PNN expression in normal adult WT brains. Here, we seek to answer whether mature PNNs are maintained as stable structures or undergo dynamic expression changes in the primary somatosensory cortex (SS1) of adult female mice after maternal experience. We focused on the SS1 due to its known roles in tactile sensation, which is also important for efficient pup retrieval (Kenyon et al., 1981; Morgan et al., 1992; Feldman and Brecht, 2005; Brecht, 2007). By using WFA as a marker and whole-brain analysis of SS1, we find that mature PNNs in adult SS1 (1) are differentially expressed in a hemisphere-specific and subregion-specific manner; (2) show dynamic expression changes after maternal experience; and (3) are influenced by MECP2, a DNA methylation reader/epigenetic regulator of chromatin and gene expression.

\section{Materials and Methods}

\section{Animals}

All experiments were performed in adult female mice (1012 weeks old) that were maintained on a 12/12 $\mathrm{h}$ light/dark cycle (lights on 07 A.M.) and received food ad libitum. Genotypes used were CBA/CaJ, Mecp2 ${ }^{\text {Het }}$ (C57BL/6 background; B6.129P2(C)-Mecp2 ${ }^{\text {tm1.1Bird } / J) ~ a n d ~ M e c p 2 ~}{ }^{W T}$-siblings (Guy et al., 2001). All procedures were conducted in accordance with the National Institutes of Health's Guide for the Care and Use of Laboratory Animals and approved by the University of Tennessee-Knoxville Institutional Animal Care and Use Committee.

\section{Pup retrieval behavior}

Pup retrieval behavior was performed as previously described (Krishnan et al., 2017; Fig. 1A). Briefly, we housed two virgin female mice (one Mecp2 ${ }^{W T}$ and one Mecp2 ${ }^{\text {Het }}$ termed "naive," NW and $\mathrm{NH}$, respectively) with a first-time pregnant CBA/CaJ female beginning 1-5 d before birth. Upon cohousing, the two naive mice are now termed Sur (SW for Sur Mecp2 ${ }^{W T}$ and SH for Sur Mecp2 ${ }^{\text {Het }}$ ). Pup retrieval behavior started on the day the pups were born (postnatal day 0; D0) as follows for each adult Sur mouse: (1) habituation phase, one adult mouse was habituated with three to five pups in the home cage for $5 \mathrm{~min}$; (2) isolation phase, pups were removed from the cage for $2 \mathrm{~min}$; and (3) retrieval phase, pups were returned to home cage, one placed at each corner and at the center (the nest was left empty if there were fewer than five pups). Each adult female had maximum of $10 \mathrm{~min}$ to gather the pups to the original nest. After testing, all animals and pups were returned to the home cage. The same procedure was performed again daily to day 5 . All behaviors were performed in the dark, during the light cycle (between 9 A.M. and 6 P.M.) and were video recorded.

\section{Immunohistochemistry}

Immediately after the behavioral trial on day 5, Sur mice as well as a set of corresponding naive Mecp2 ${ }^{W T}$ and Mecp2 ${ }^{\text {Het }}$ mice were perfused with $4 \%$ paraformaldehyde/PBS, and brains were extracted and postfixed overnight at $4^{\circ} \mathrm{C}$. Brains were then treated with $30 \%$ sucrose/ PBS overnight at room temperature (RT) and sectioned in sagittal orientation using a freezing microtome at $70 \mu \mathrm{m}$. Free-floating brain sections were immunostained at RT as previously described in Krishnan et al. (2017), with a few modifications. Briefly, sections were blocked in $10 \%$ normal goat serum and $0.5 \%$ Triton X-100 for $3 \mathrm{~h}$, then incubated with biotin-conjugated WFA Lectin (labels PNNs; 1:500; Sigma-Aldrich) and rabbit MECP2 primary antibody (1:1000; Cell Signaling) overnight at RT in a 5\% normal goat serum and $0.25 \%$ Triton X-100 solution. Then, sections were incubated for $4 \mathrm{~h}$ with Alexa Fluor 488 and Texas-Red secondary antibodies (1:1000; Invitrogen) in a $5 \%$ normal goat serum and $0.25 \%$ Triton $X-100$ solution. Finally, sections were counterstained with the nuclear marker, DAPI (1:1000) for $5 \mathrm{~min}$, and mounted in Fluoromount-G (Southern Biotech).

\section{Image acquisition and analysis}

To analyze PNNs, $10 \times$ single-plane PNN images of the entire SS1 from each brain slice were acquired on a motorized stage, epifluorescent microscope (Keyence BZX710; Keyence Corp.) and stitched using BZ-X Analyzer (Keyence; Fig. 1B). Our initial observation of PNN intensity showed that $\mathrm{SH}$ had the most intense fluorescent signal in SS1. Thus, imaging settings were established based on $\mathrm{SH}$ within each cohort of mice, to minimize overexposure. The light exposure time for fluorescent signal acquisition was identified by finding the exposure time where a saturated pixel first appears within frame, then decreasing the exposure time by 1 unit, according to software specifications. This exposure time determination method was applied to all brain sections of $\mathrm{SH}$ in each cohort and the mean exposure time was calculated and used for final image acquisition within each cohort.

For PNN image analysis, each stitched image was opened in ImageJ (Schneider et al., 2012). Then, SS1 and somatosensory subregion areas were (1) mapped by overlaying templates from Paxinos and Franklin's The Mouse Brain, fourth edition (Paxinos and Franklin, 2013), (2) outlined, and (3) measured using the functions in ImageJ. To count high-intensity mature PNNs, the Contrast setting from the browser was set to the far right to threshold weaker signals. The remaining signals were manually quantified under the classification that a "mature" PNN is at least $80 \%$ of its original shape (before contrast adjustment). Detailed protocol for mapping and 
A
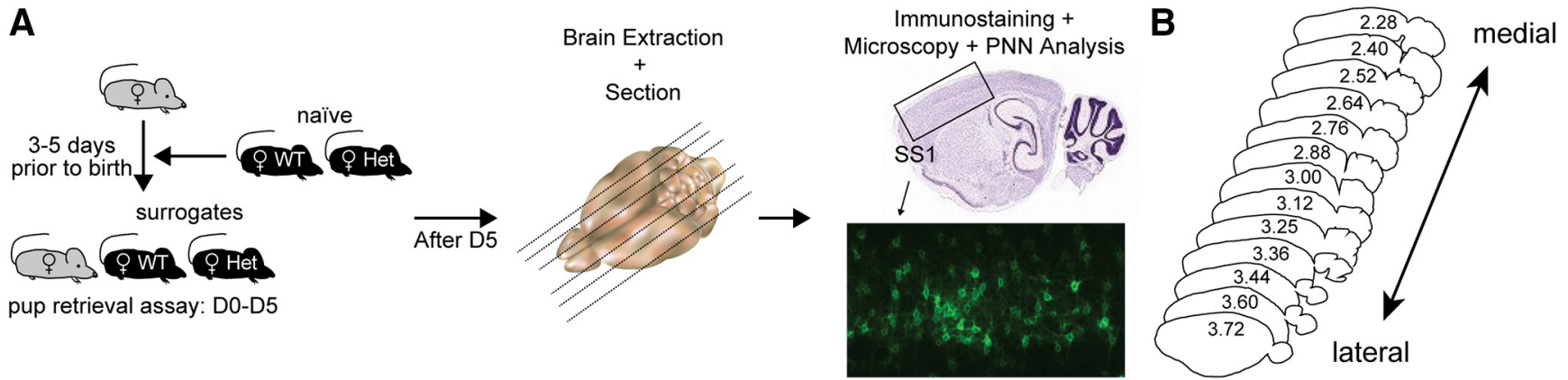

Figure 1. Schema representing behavioral and histology pipeline. (A) (Left) Alloparental behavioral model in mice. Pregnant CBA CaJ female (grey mouse) is cohoused with adult female naïve WT and naïve Het littermate controls, which changes their status to surrogates, 3-5 days before birth of pups. Once pups are born, pup retrieval assay is performed with the surrogates from day 0 (D0) to 5 (D5). (Center) After behavioral experiments on D5, surrogate mice and age-matched naïve counterparts are perfused, their brains extracted, and sectioned as a single cohort. (Right) Standard immunostaining and imaging with epifluorescent slide scanner are performed, to image and analyze PNNs, as elaborated in Methods. (B) Schema of mouse brain sections cut in sagittal orientation, depicting all medial $(2.28 \mathrm{~mm}$, corresponding to map number 120$)$ to lateral $(3.72 \mathrm{~mm}$, corresponding to map number 132$)$ regions of SS1 analyzed for this study. Coordinate maps are based on Paxinos and Franklin's mouse brain atlas, $4^{\text {th }}$ edition.

counting procedures are now available at dx.doi.org/ 10.17504/protocols.io.bcf8itrw. All statistical analyses and graphs were generated using GraphPad Prism.

For MECP2 expression analysis, a portion of SS1 region was imaged using $20 \times$ objective and Keyence microscope, with tiled and stacks of 11 images, which were then collapsed and stitched using the Keyence stitch function. Imaging settings were based on left hemisphere of one brain and applied to the right hemisphere of the same brain. Measurement of MECP2 intensity was perform using ImageJ as follows: (1) for each tiled projection image, a region of interest (ROI) box of $1.5 \mathrm{~mm}$ (rostralcaudal) $\times 0.5 \mathrm{~mm}$ (dorsal-ventral) to select a large portion of SS1, which covers Layers II/III-V where most of the PNNs are localized; (2) individual intensity values were acquired and saved by selecting Analyze, Tools and Save $X Y$ Coordinates; and (3) exported Excel values were sorted and percentage cumulative frequency distribution were calculated using Microsoft Excel and GraphPad Prism (version 8).

\section{Principal component analysis (PCA) on PNN density}

We used PNN densities (counts per area) for the entire SS1 across all individuals in the five cohorts. If multiple sections per map number were present, values were averaged across sections to give a single density. In the first PCA, to determine whether the PNN patterns segregated primarily by cohort or (genotype and experience), we kept the data for each individual animal separate and averaged PNN densities across every set of two adjacent map regions. Because the scale of raw numbers varied so widely between individual animals, we next median normalized PNN densities within each animal. To do this, we calculate the median PNN density across all map numbers for each individual. Then, each individual PNN density for each map number of that individual was divided by ("normalized by") this median. Finally, these median normalized densities were averaged across all individuals in the same condition. This method of normalization accounts for the variability in the median across cohorts within the same condition. Some regions for some individuals had no data, and, because PCA does not tolerate missing data, we imputed these missing values by using the average median normalized values for that region from animals in other cohorts in the same condition. By running PCA in R, we obtained a weight for each map number showing the highest variance patterns in PNN densities across the individual animals. We then calculated the projection of each animal onto principle components 1 and 2. K-means clustering was used to calculate the two most evident clusters in this PCA space.

In the second PCA, to determine the major PNN density patterns that distinguish genotypes and experience conditions, we left data for each map number separate, but averaged the median normalized PNN densities across the five cohorts for each condition. We then performed PCA to determine the major patterns that distinguish these conditions.

\section{Results}

\section{PNN density changes across somatosensory cortical maps}

As somatosensation during pup retrieval primarily involves facial/snout areas in Sur (non-lactating adult females; Morgan et al., 1992; Stern, 1996; Lonstein and Stern, 1997), we present data collected from the somatosensory cortical regions involved in processing tactile stimuli in the alloparental care paradigm (Fig. 1A). According to Paxinos and Franklin's atlas (fourth edition), there are eight different anatomic somatosensory cortical subregions [S1; S1 barrel field (S1BF), S1 dysgranular zone (S1DZ), S1 fore limb (S1FL), S1 jaw (S1J), S1 upper lip (S1ULp), S1 trunk (S1Tr), S1 hindlimb (S1HL) and undefined somatosensory cortex S1], here collectively called SS1 (Paxinos and Franklin, 2013). To determine how PNN expression changes across the different SS1 

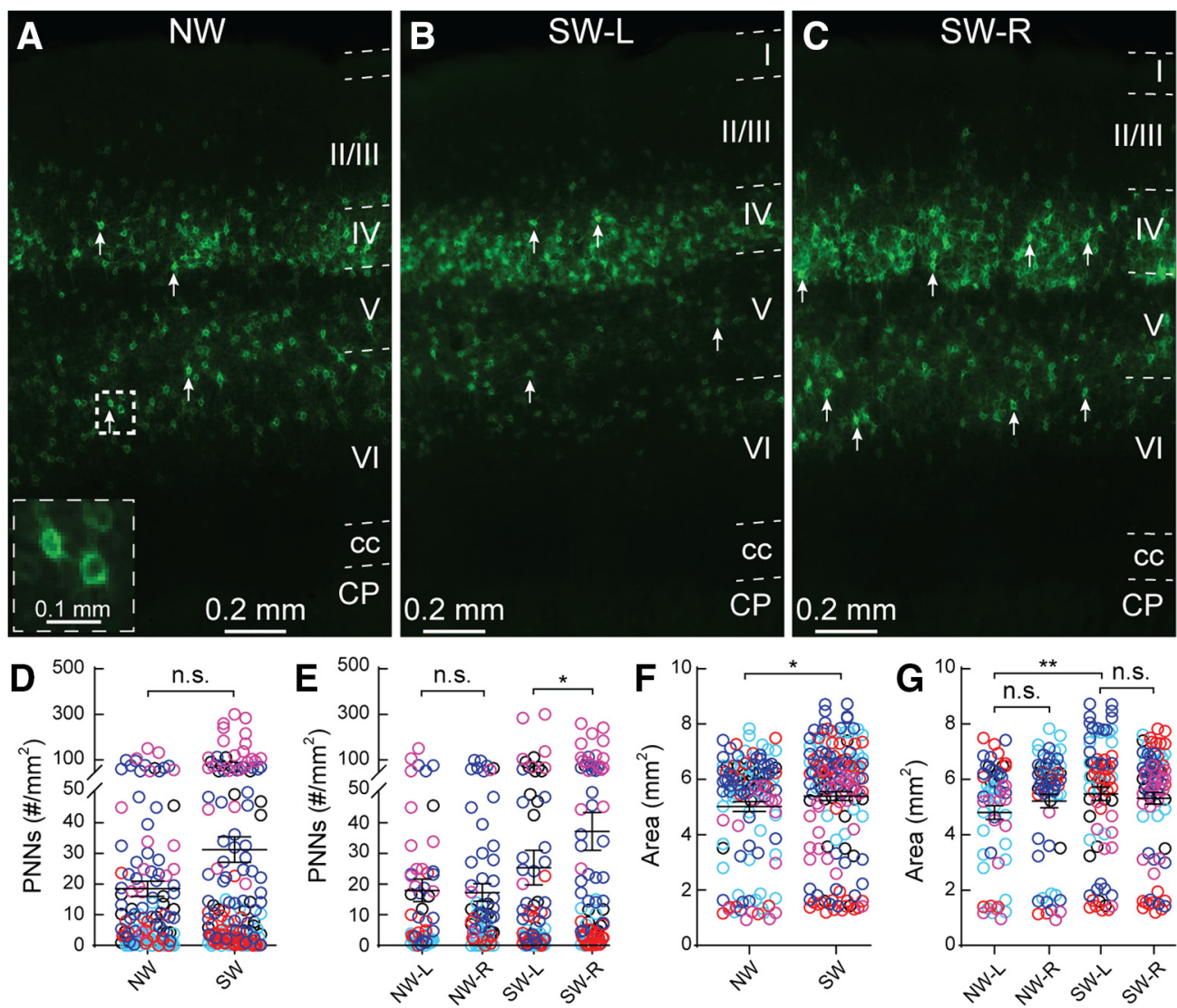

Figure 2. Surrogate $M e c p 2^{W T}$ (Wild type) mice exhibit hemisphere specific increase in PNN density in the primary somatosensory cortex (SS1). (A-C) Representative epifluorescent images of PNN expression in SS1 of naïve WT (NW) (A) as well as left (L) (B) and right $(R)(C)$ hemispheres of surrogate WT (SW). Layers 1 through 6 are outlined. CP = caudate putamen and cc $=$ corpus callosum. Arrows indicate examples of high-intensity PNNs analyzed for the study. Inset in A shows magnified PNN structures in the box. (D) Combined hemisphere analysis of the density of high-intensity PNNs was not significantly different between NW and SW (NW: $n=$ 123 images; SW: $n=162$; Mann-Whitney test, $p>0.05)$. (E) Separate analysis of left and right hemisphere revealed a significant increase of high-intensity PNNs in the right hemisphere of SW (SW-R; $n=81$ images) compared to the left hemisphere of SW (SW-L; $n=81$ images) (Kruskal-Wallis followed by Dunn's test, $* p<0.05)$, while no significant difference was observed between hemispheres of NW (NW-L: $\mathrm{n}=59$ images; NW-R: $n=64$ images; Mann-Whitney test, $p>0.05$ ). (F,G) Size of SS1 was overall significantly larger in SW compared to NW (F) (NW: $n=123$ images; SW: $n=162$ images; Mann-Whitney test, $* p<0.05$ ). Enlargement of SS1 in SW occurred in the left hemisphere (G) (NW-L: $n=59$ images, NW-R: $n=64$ images; SW-L: $n=81 \mathrm{images;}$ SW-R: $n=81 \mathrm{im}-$ ages; Kruskal-Wallis followed by Dunn's test, $* * p<0.01$ ). For D-G, n.s. = not significant. Different colors represent each of the five cohorts. Each open circle represents PNN density in an individual brain section.

subregions, we took a systematic approach covering the whole SS1, rather than the standard approach of analyzing "representative sample sections" (Fig. 1B). We analyzed $40-60$ sagittal brain sections (at $70 \mu \mathrm{m}$ each, both left and right hemispheres) per animal, in five biological replicates across four conditions [naive WT (NW), naive Het (NH), Sur WT (SW), Sur Het (SH); Fig. 1A], as qualitative regional differences in PNN density were observed in pilot studies in our lab. The SS1 subregions are represented by map numbers 120-132, corresponding to lateral coordinates $2.28 \mathrm{~mm}$ (medial region) to $3.72 \mathrm{~mm}$ (lateral region), which encompass $\sim 1.5 \mathrm{~mm}$ of one mouse brain hemisphere.

PNN expression is predominantly found in deeper Layers IV, V, and VI in the adult SS1 in both NW (Fig. 2A) and SW mice (Fig. 2B,C). Across the five cohorts, PNN density (as measured by high-intensity PNN counts over area) was not significantly different between NW and SW across the whole SS1 (Fig. 2D). However, we noticed a wide range of distribution of PNN density within individual animals across the cohorts (Fig. 2D). In order to determine the source of such variability, we parsed the data according to hemispheres (Fig. 2E), across the lateral-medial axis (Fig. 3) and by subregions (Fig. 4). No significant difference was observed in PNN density between the left and right hemispheres of NW (Fig. 2E); however, the right hemisphere of SW (SW-R) had higher PNN density than the left hemisphere (SW-L; Fig. 2B,C,E), suggesting that higher PNN density in the SW-R SS1 might consolidate new tactile information related to the pups and/or the mother.

PNN density is PNN expression counts over area. Overall, SS1 area of the SW is significantly increased, compared with NW (Fig. 2F); however, that increase was 

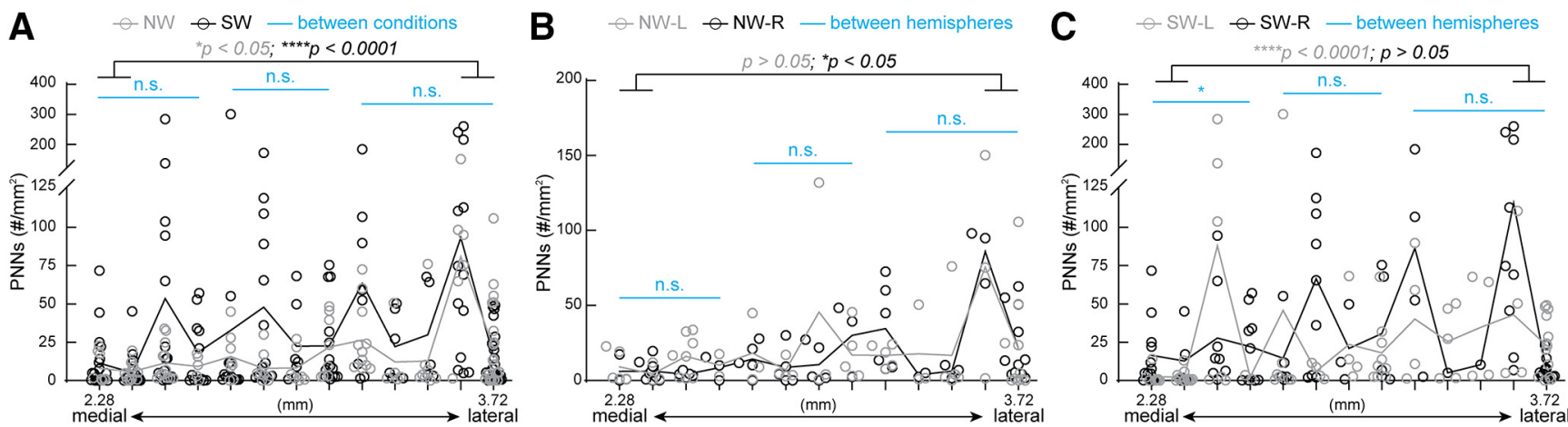

Figure 3. Dynamic changes in PNN density across medial-lateral axis of both naïve and surrogate WT SS1. (A) Distribution of combined PNN density from left and right hemispheres of SS1 revealed an increase of PNN density in lateral sections compared to the medial sections of the same condition. Statistical analysis revealed significant differences between the combined two most medial regions and the combined two most lateral regions within conditions (grey $p$ value denotes NW: $n=22$ images for medial, 28 images for lateral; black $p$ value denotes SW: $n=37$ images for medial, 37 images for lateral). This medial-lateral comparison analysis method also applies to all subsequent figure panels. No significant difference in PNN density was found between conditions, represented by the light blue lines. Statistical analysis of the most medial region was a combined of 4 map numbers (NW: $n=40$ images; SW: $n=62$ images), middle region was a combined of 4 map numbers (NW: $n=32$ images; SW: $n=46$ images) and most lateral region was a combined of 5 map numbers (NW: $n=52$ images; SW: $n=56$ images). This sub-regional comparison method also applies to all subsequent figure panels. $N=5-24$ images per map number. (B) Analysis of the right hemisphere in NW revealed that lateral sections had significantly higher density than the medial sections (NW-R, black $p$ value; $n=14$ images for medial, 15 images for lateral). In the left hemisphere, NW-L did not show any significant difference in medial-lateral axis (grey $p$ value; $n=8$ images for medial, 13 images for lateral). Across the subregions in the medial-lateral axis, there was no significant difference between the hemispheres in NW (blue lines; medial-NW: $n=18$ images for L, 22 images for R; middle-NW: $n=15$ images for L, 18 images for R; lateral-NW: $n=26$ images per hemisphere). (C) A different pattern of hemisphere-specific differences was observed in SW. SW-R did not have significant differences between medial and lateral sections, while SW-L did (grey $p$ value denotes SW-L: $n=20$ images for medial, 17 images for lateral; black $p$ value denotes SW-R: $n=17$ images for medial, 20 images for lateral). While PNN density did not differ between SW-L and SW-R in middle and lateral regions, SW-R exhibited significantly higher PNN density in the medial regions than SW-L (medial-SW: $n=28$ images for L, 34 images for R; middle-SW: $n=24$ images for L, 22 images for R; lateral-SW: $n$ $=30$ images for L, 26 images for R). For A-C, lines represent the mean values. Each dot represents PNN density in an individual section. Mann-Whitney test: $* p<0.05$, n.s. = not significant, 5 mice per condition. For $\mathrm{B}$ and $\mathrm{C}, n=1-13$ images per map number.

not specific to a particular hemisphere within experimental condition (NW-L vs NW-R; SW-L vs SW-R; Fig. 2G). There is a small but significant area increase in SW-L, compared with left hemisphere of NW (Fig. 2G). Thus, the increased PNN density in the SW-R is mainly due to the PNN expression, and not due to the change in area.

Next, we plotted PNN density in individual sections across the lateral-medial axis (Fig. 3). We observed the individual variability in sections across map numbers in both NW and SW (Fig. 3A). In line with Figure 2D, we did not see any significant differences between NW and SW in the medial-lateral axis (light blue lines across multiple map number subdivisions). However, PNN density is significantly higher in the two most lateral sections compared with the two most medial sections within conditions (gray $p$ value for NW, black $p$ value for SW). Furthermore, NW had similar PNN density between left (gray) and right (black) hemispheres across the medial-lateral axis (Fig. $3 B$, light blue lines), with the more lateral regions of right NW having slightly but significantly higher PNN density compared with its medial regions (black $p$ value). SW showed many sections across the medial-lateral axis with higher PNN density in the right hemisphere (black) than the left hemisphere (gray), particularly in the most medial sections (Fig. 3C, blue line). Within hemisphere, lateral regions of SW-L had significantly higher PNN density than its medial regions (gray $p$ value), while there was no difference between these regions in SW-R (black $p$ value). These findings indicate differential PNN expression in a position-specific, hemisphere-specific manner in the SS1 in both NW and SW.

\section{Changes in PNN density are SS1 subregion specific}

Next, we examined whether specific subregions of SS1 were particularly plastic for PNN density between NW and SW. We observed no significant differences in PNN density in subregions S1BF, S1DZ, and S1FL when aggregating both hemispheres (Fig. 4A-C). For S1BF, a region well studied for whisker activity that contributes to tactile sensation, PNN density increased significantly and specifically in the right hemisphere for SW, compared with NW (Fig. 4A'). This result suggests that increased PNN density in the right hemisphere of S1BF could be a potential site for consolidation of tactile sensory information relevant for executing efficient pup retrieval. Contrary to the pattern in S1BF, S1DZ, and S1FL regions showed increased $P N N$ density in the SW-R, compared with its SW-L (Fig. 4B', C'). S1DZ has been implicated in proprioceptive functions, such as the movement of joints and stretch of muscle receptors (Chapin and Lin, 1984; Welker et al., 1984; Lee and Kim, 2012; Shin Yim et al., 2017); while S1FL is the sensory 

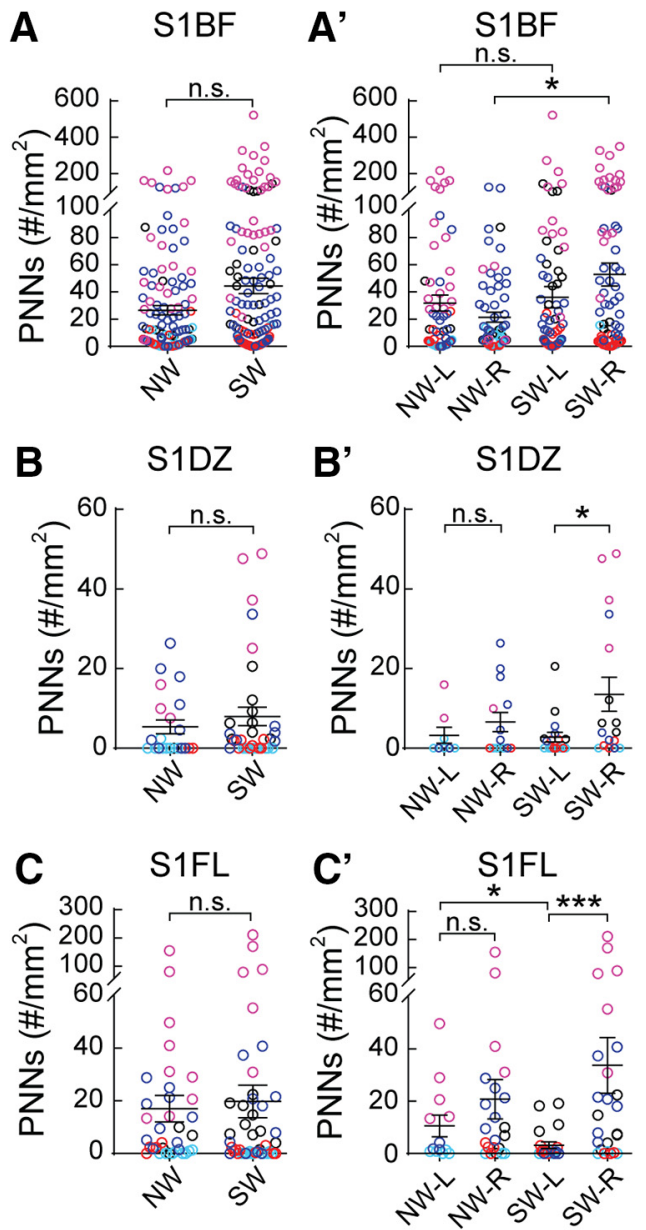

Figure 4. PNN density varies across subregions of WT SS1. $(\boldsymbol{A}-\boldsymbol{C})$ Analysis of both hemispheres for SS1 barrel field (S1BF, A), dysgranular zone (S1DZ, B) and forelimb (S1FL, C) revealed no significant differences between NW and SW (S1BF: NW $-n=131$ images, SW $-n=168$ images; S1DZ: NW $-n=22$ images, SW $-n=35$ images; S1FL: NW $-n=$ 35 images, SW $-n=46$ images; 5 mice per condition; Mann-Whitney test, $p>0.05$, n.s. = not significant). $\left(\boldsymbol{A}^{\prime}-\boldsymbol{C}^{\prime}\right)$ Analysis of sub regional SS1 by hemispheres revealed dynamic changes in PNN expression. In S1BF $\left(\boldsymbol{A}^{\prime}\right)$, a significant increase of PNN expression was detected in the right hemisphere of SW (SW-R) compared to NW (NW-R) (NW-L: $n=65$ images; NW-R: $n=66$ images; SW-L: $n=85$ images; SW-R: $n=83$ images). In S1DZ $\left(\boldsymbol{B}^{\prime}\right)$, while no significant difference was observed in PNN density between hemispheres in NW, there was a significant increase of PNN density in the right hemisphere (SW-R) compared to the left (SW-L) (NW-L: $n=8$ images; NW-R: $n=14$ images; SW-L: $n$ $=18$ images; SW-R: $n=17$ images). A similar pattern of PNN plasticity was also detected in S1FL $\left(\boldsymbol{C}^{\prime}\right)$, where PNN density was significantly increased in the right hemisphere than the left of SW. Interestingly, SW-L exhibited significantly lower PNN density compared to NW-L (Figure 4C'; NW-L: $n=13$ images; NW-R: $n=22$ images; SW-L: $n=21$ images; SW-R: $n=25$ images). For $\boldsymbol{A}^{\prime}-\boldsymbol{C}^{\prime}, 5$ mice per condition; Kruskal-Wallis followed by Dunn's test, ${ }^{*} p<0.05,{ }^{* * *} p$ $<0.001$, n.s. $=$ not significant . representation of the forepaw. Currently, the roles of these regions and PNN contribution in maternal behavior is unclear. Other brain regions (S1ULp, S1J, and S1) showed no changes after surrogacy, which further highlights S1BF as a potential site for learning consolidation (Table 1). In analyzing left hemisphere-specific data, there was a significant decrease in PNN density in S1FL of SW compared with NW (Fig. 4C'; Table 1, columns 5, 7). This is the first report, to our knowledge, of decreases in PNN density in a social behavior context in adult brains. Together, these results suggest that PNN density changes in adult females, in a hemisphere-specific and subregion-specific manner that is conducive for experience-dependent plasticity.

\section{Appropriate PNN expression in SS1 is dependent on MECP2}

MECP2 is thought to regulate experience-dependent plasticity mechanisms in an epigenetic manner, in early postnatal development and in adulthood (Guy et al., 2001; Dani et al., 2005; Muotri et al., 2010; Cohen et al., 2011b; Noutel et al., 2011; Durand et al., 2012; Gabel et al., 2015; Krishnan et al., 2015, 2017; Lagger et al., 2017; Morello et al., 2018; Picard and Fagiolini, 2019). We previously tested this hypothesis, using an alloparental care paradigm, and found that Mecp2-Het adult females were inefficient at pup retrieval (Krishnan et al., 2017). In this study, we identified atypical and transient increases in PNN density in the auditory cortex of $\mathrm{SH}$, leading to altered responses of $\mathrm{PV}+$ neurons to auditory cues in $\mathrm{SH}$ (Lau et al., 2020). Here, we sought to determine whether SS1 of SH exhibited similar alterations in PNN density in subregion-specific ways. Comparing $\mathrm{NH}$ to $\mathrm{SH}$, we noticed no significant differences in PNN density in whole SS1 (Fig. $5 A$, similar to WT in Fig. $2 D$ ), or within hemispheres of SS1 (Fig. 5B, unlike WT in Fig. 2E). There were no significant changes in SS1 area between $\mathrm{NH}$ and SH (Fig. 5C, unlike SW in Fig. 2F). However, an increased SS1 area in left versus right hemisphere of $\mathrm{SH}$ was noted (Fig. 5D).

For PNN density analysis across medial-lateral axis (Fig. 6), PNN density was similar between $\mathrm{NH}$ and $\mathrm{SH}$ along this axis (Fig. 6A, light blue lines), with significantly more PNNs in the lateral regions than the medial regions within conditions (gray $p$ value for $\mathrm{NH}$, black $p$ value for $\mathrm{SH}$ ). Within $\mathrm{NH}$, there was no difference between left and right hemispheres along medial-lateral axis (Fig. 6B, light blue lines). Again, lateral regions expressed significantly more PNNs than the medial regions within each hemisphere (gray $p$ value for $\mathrm{NH}-\mathrm{L}$, black $p$ value for $\mathrm{NH}-\mathrm{R}$ ). After surrogacy, $\mathrm{SH}$ exhibited significantly more dynamic changes in PNN density across medial-lateral axis between left and right hemisphere (Fig. 6C, light blue lines). Moreover, while PNN densities in lateral and medial regions were not significantly different in SH-R (black $p$ value), lateral regions of $\mathrm{SH}-\mathrm{L}$ had significantly more PNNs than the its medial regions (gray $p$ value).

When we compared PNN density between genotypes, WT and Het (Table 1; Fig. 7), we noticed significant hemisphere-specific and subregion-specific differences, with 
Table 1: Average high-intensity PNN density across subregions and hemispheres of the SS1 before and after maternal behavior experience

\begin{tabular}{|c|c|c|c|c|c|c|c|c|c|c|c|c|}
\hline \multirow[t]{2}{*}{ Regions } & \multicolumn{4}{|c|}{ Whole brain } & \multicolumn{4}{|c|}{ Left hemisphere } & \multicolumn{4}{|c|}{ Right hemisphere } \\
\hline & NW & $\mathrm{NH}$ & SW & $\mathrm{SH}$ & NW-L & $\mathrm{NH}-\mathrm{L}$ & SW-L & SH-L & NW-R & $\mathrm{NH}-\mathrm{R}$ & SW-R & SH-R \\
\hline S1BF & $26.6 \pm 3.5^{\circ}$ & $56.0 \pm 6.6^{\oplus}$ & $44.4 \pm 5.8$ & $47.0 \pm 5.4$ & $31.7 \pm 5.9^{f}$ & $41.0 \pm 5.9^{f}$ & $36.1 \pm 7.8$ & $37.6 \pm 5.4$ & $21.4 \pm 3.6^{g, h}$ & $72.2 \pm 11.9^{9}$ & $52.8 \pm 8.4^{\mathrm{h}}$ & $56.3 \pm 9.3$ \\
\hline S1ULp & $28.3 \pm 4.8^{\circ}$ & $47.0 \pm 8.1^{\circ}$ & $37.9 \pm 5.7$ & $52.9 \pm 7.6$ & $33.4 \pm 7.3$ & $45.3 \pm 8.2$ & $29.1 \pm 6.7$ & $44.2 \pm 9.7$ & $23.5 \pm 6.3$ & $49.2 \pm 15.2^{p}$ & $38.9 \pm 8.0$ & $62.5 \pm 11.8^{p}$ \\
\hline S1FL & $17.0 \pm 5.0$ & $20.9 \pm 4.0$ & $19.8 \pm 6.2$ & $18.4 \pm 3.6$ & $10.6 \pm 4.2^{3}$ & $20.8 \pm 5.0$ & $3.2 \pm 1.3^{s, t, u}$ & $8.1 \pm 2.2^{t, v}$ & $20.8 \pm 7.5$ & $21.0 \pm 6.4$ & $33.8 \pm 10.7^{\mathrm{u}}$ & $30.6 \pm 6.6^{v}$ \\
\hline S1J & $2.5 \pm 0.8^{i}$ & $4.2 \pm 0.9^{i}$ & $6.7 \pm 3.6$ & $8.4 \pm 2.0$ & $4.0 \pm 1.6^{k}$ & $5.2 \pm 1.4^{k,}$ & $9.0 \pm 6.8$ & $3.6 \pm 1.2^{1}$ & $1.3 \pm 0.4^{\mathrm{m}}$ & $3.0 \pm 0.7^{\mathrm{m}}$ & $4.2 \pm 1.3^{n}$ & $14.0 \pm 3.9^{n}$ \\
\hline S1 & $6.5 \pm 0.9^{\mathrm{a}}$ & $16.7 \pm 2.8^{\mathrm{a}}$ & $14.4 \pm 3.7^{b}$ & $14.4 \pm 2.1^{b}$ & $7.0 \pm 1.6$ & $11.4 \pm 2.5$ & $16.7 \pm 6.6$ & $11.4 \pm 2.1$ & $6.0 \pm 1.0^{c}$ & $22.5 \pm 5.0^{\circ}$ & $12.3 \pm 3.2^{d}$ & $17.5 \pm 3.6^{d}$ \\
\hline S1DZ & $5.4 \pm 1.7$ & $9.1 \pm 2.6$ & $8.0 \pm 2.3$ & $12.7 \pm 3.5$ & $3.2 \pm 2.0$ & $7.4 \pm 3.4$ & $2.8 \pm 1.2^{q}$ & $6.7 \pm 4.0^{r}$ & $6.6 \pm 2.4$ & $10.1 \pm 3.8$ & $13.4 \pm 4.3^{q}$ & $20.6 \pm 5.6^{r}$ \\
\hline
\end{tabular}

NW, NH, SW and SH are the four different conditions. Primary somatosensory cortex subregions: S1BF, S1ULp, S1FL, S1J, S1, and S1DZ. Significant differences are denoted between genotypes by shading (E.g., NW vs NH), between hemispheres of the same condition by bold lettering, and between Naïve and Surrogate in the same genotype by bold borders. Each letter pair corresponds to statistically significant differences between two conditions. Numbers correspond to average PNN density with standard error mean across multiple sections. N = 8-85 images for hemisphere analysis, $123-168$ images for combined hemisphere analysis; 5 mice per condition; Kruskal-Wallis followed by Dunn's test.

the maintenance of medial-lateral axis. $\mathrm{NH}$ had increased PNN density over NW across medial-lateral axis (Fig. 7A) and significant differences between genotypes after surrogacy (SW vs $\mathrm{SH}$ ) in lateral sections (Fig. 7D, light blue line). S1 was an exception, which showed statistical significance, though the mean density was similar (Table 1). This is likely due to differential distribution of PNN density (Fig. 8).

In the left hemisphere, $\mathrm{NH}$ and $\mathrm{SH}$ (Fig. 7B,E, respectively) had significantly higher PNN density in the lateral sections, compared with NW and SW, respectively. No significant differences were observed between genotypes in the right hemisphere (Fig. 7C,F). While analyzing subregions, in the left hemisphere, $\mathrm{NH}$ had increased PNN density in S1BF and S1J, compared with NW (Table 1, columns 5, 6). SH had increased PNN density in only S1FL, compared with SW (Table 1, columns 7, 8). In the right hemisphere, $\mathrm{NH}$ had increased PNN density in S1BF, S1J, and S1, compared with NW. SH had increased PNN density in
S1J and S1, compared with SW. Surrogacy correlated with higher PNN density in only right hemisphere of WT S1BF (NW-R vs SW-R; also Fig. 2E), while this was not seen in Het (NH vs $\mathrm{SH}$ ), with $\mathrm{NH}-\mathrm{R}$ already exhibiting high PNN densitycompared with NW-R (Table 1, columns 9, 10). S1ULp showed higher PNN density in SH$\mathrm{R}$ compared with $\mathrm{NH}-\mathrm{R}$ (Table 1, columns 10, 12), which was not observed in WT. Taken together, these results suggest that MECP2 regulates dynamic PNN expression, which is then important for appropriate maternal behavior.

Comparing the hemispheres within genotypes, NW and $\mathrm{NH}$ did not have significant differences in PNN density in subregions. However, PNN density increased in the right hemisphere of S1FL and S1DZ in both SW and SH (Table 1) compared with $S W-L$ and $S H-L$, respectively. These results suggest a preserved common experience-dependent plasticity mechanism activation in these regions within the right hemisphere across genotypes in similar social contexts.
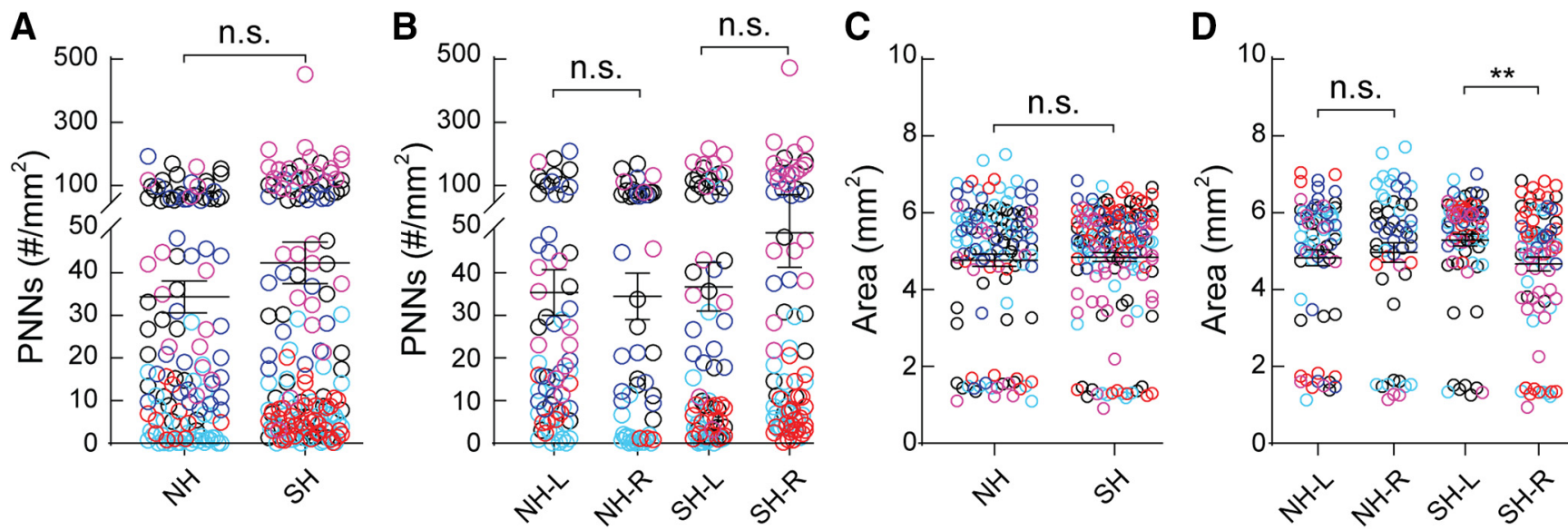

Figure 5. Hemisphere-specific PNN density changes are not conserved in Mecp2 ${ }^{\text {Het }}$ after maternal behavior. (A) Combined hemispheric analysis of PNN density of SS1 did not reveal significant changes between naïve Het (NH, $n=123$ images) and surrogate Het (SH, $n=162$ images) (Mann-Whitney test, $p>0.05)$. (B) Analysis of PNN density between hemispheres of SS1 revealed no significant difference between conditions or within hemispheres of naïve or surrogate Het $(n=54-82$ images; Kruskal-Wallis followed by Dunn's test, $p>0.05)$. (C) Analysis of SS1 area in both hemispheres revealed no significant changes after maternal learning ( $\mathrm{NH}$ : $n=123$ images; $\mathrm{SH}: n=162$ images; Mann-Whitney test, $p>0.05)$. (D) Area analysis by hemispheres reveal that left hemisphere of $\mathrm{SH}(\mathrm{SH}-\mathrm{L})$ was significantly larger than the right hemisphere of $\mathrm{SH}(\mathrm{SH}-\mathrm{R})$. This hemispheric area bias was absent in $\mathrm{NH}(n=54-82$ images; Kruskal-Wallis followed by Dunn's test, $\left.{ }^{* *} p<0.01\right)$. n.s. = not significant. 5 mice per condition. 

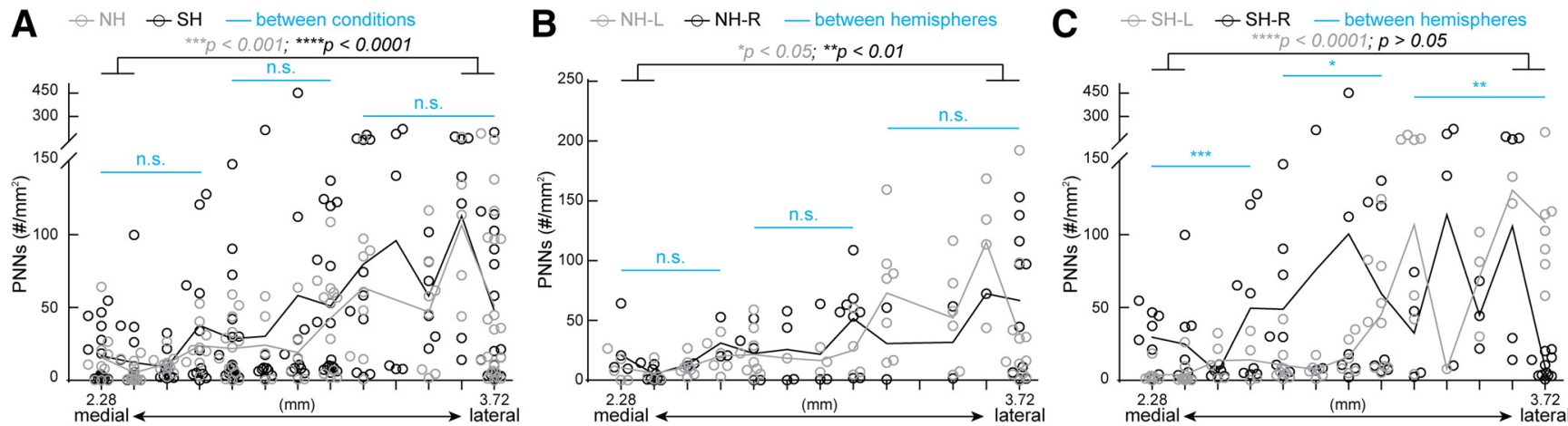

Figure 6. Overall patterns of PNN density across medial-lateral axis is preserved in Mecp $2^{H e t}$. (A) Similar to NW-SW in Figure 3 , NH and $\mathrm{SH}$ displayed significant increase of PNN density in lateral sections compared to the medial sections of the same condition (grey $p$ value denotes $\mathrm{NH}: n=23$ images for medial, 27 images for lateral; black $p$ value denotes SH: $n=36$ images for medial, 25 images for lateral). Along the sub regions encompassing the medial-lateral axis, there was no significant difference between $\mathrm{NH}$ and $\mathrm{SH}$, represented by the light blue lines (medial: $n=42$ images for $\mathrm{NH}, 59$ images for SH; middle: $n=37$ images for $\mathrm{NH}$, 55 images for SH; lateral: $n=44$ images for $\mathrm{NH}, 48$ images for $\mathrm{SH}$ ). $N=5-22$ images per map coordinate. (B) Within hemispheres of NH, statistical analysis of the lateral sections of the right ( $\mathrm{NH}-\mathrm{R}$, black $p$ value) and the left $(\mathrm{NH}-\mathrm{L}$, grey $p$ value) showed significantly higher PNN density than their medial sections (NH-L: $n=9$ images for medial, 16 images for lateral; NH-R: $n=14$ images for medial, 11 images for lateral). There was no significant difference between hemispheres along the medial-lateral axis (medial: $n=23$ images for $\mathrm{L}$, 19 images for R; middle: $n=17$ images for L, 20 images for R; lateral: $n=29$ images for L, 15 images for R). (C) A different pattern of hemisphere-specific differences was observed in $\mathrm{SH}$. SH-R did not display significant difference between medial and lateral sections, while SH-L did (grey $p$ value denotes SH-L: $n=9$ images in lateral, $n=20$ images for medial; black $p$ value denotes SH-R: $n=$ 16 images for both lateral and medial). Statistical comparison between hemispheres revealed dynamic differences across mediallateral axis, with SH-R had significantly higher PNN density than SH-L in medial and middle regions, while SH-L had significantly higher PNN density in the lateral regions (light blue lines; medial: $n=29$ images for L, 30 images for R; middle: $n=31$ images for L, 24 images for R; lateral: $n=20$ images for L, 28 images for R). For $\boldsymbol{A}-\boldsymbol{C}$, lines represent mean values. Each dot represents PNN density in an individual section. Mann-Whitney test: ${ }^{*} p<0.05,{ }^{* \star} p<0.01,{ }^{* \star *} p<0.001,{ }^{* \star * *} p<0.0001$, n.s. $=$ not significant, 5 mice per condition. For $\boldsymbol{B}-\boldsymbol{C}, n=1-12$ images per map coordinate.

\section{PCA identifies lateral-medial and hemisphere-specific changes in PNN expression}

Because of the increasing number of variables being compared, we chose an unsupervised statistical procedure called Principal Component Analysis (PCA), commonly used in genomics/transcriptomics analysis, to determine whether patterns emerge from the PNN density data. PCA takes a set of measurements across samples and identifies the measurements that best capture the variation among the samples. It results in a set of uncorrelated components (called principal components) that each capture an orthogonal aspect of the differences across the samples. As input to PCA, we used PNN densities across individual sections and map numbers (represented as lateral coordinates) across all conditions in the five cohorts. If multiple sections per map number were present, values were averaged across sections to give a single density value.

In the first analysis (Fig. 9A,B), we sought to determine whether the PNN patterns segregated primarily by cohort or condition (genotype and experience). We preserved data for each individual brain and performed PCA on PNN densities averaged across every set of two adjacent map regions. By examining the projection of each individual onto the first and second principal components, we found that, while there is biological variability between cohorts, the individuals in a given cohort did not cluster separately from one another in this unsupervised analysis (Fig. 9A, left), suggesting that technical variability in processing samples across five cohorts is not the primary driver of
PNN density differences. This is an important control to assess technical or biological variability in this data. Instead, the first principal component (PC1), which explains $40 \%$ of the variation in the data (Fig. $9 A$, right, inset), distinguished the SH PNN density patterns from all others, especially $\mathrm{NH}$ (Fig. 9A, right). We then examined the weights of each brain region in PC1 to determine which regions are most important for capturing the differences between $\mathrm{SH}$ and $\mathrm{NH}$. The weights of each section in PC1 (and PC2) shows a left-right asymmetry and increasing weights for lateral versus medial sections, confirming our previous observations (Fig. 9B). The findings from our first PCA confirms that variations among mice are resulting from genetic and/or environmental differences and not technical biases. The results further validate our previous observations of asymmetry in PNN density of left and right hemispheres, augmentation of PNN density in lateral sections, and altered SH PNN density patterns.

In the second PCA (Fig. 9C,D), we sought to determine the major PNN density patterns that distinguish genotypes and conditions. Instead of averaging map numbers as in the previous analysis, we preserved data for each map number and averaged across the five cohorts for each condition and then performed PCA to determine the major distinguishing patterns. This analysis revealed patterns of PNN density that best distinguish $\mathrm{NH}$ versus $\mathrm{SH}$ (PC1) and SW versus SH (PC2; Fig. 9C,D). These PC patterns also reflect the medial/lateral and left/right asymmetries, thus confirming the anatomic and neurobiological 

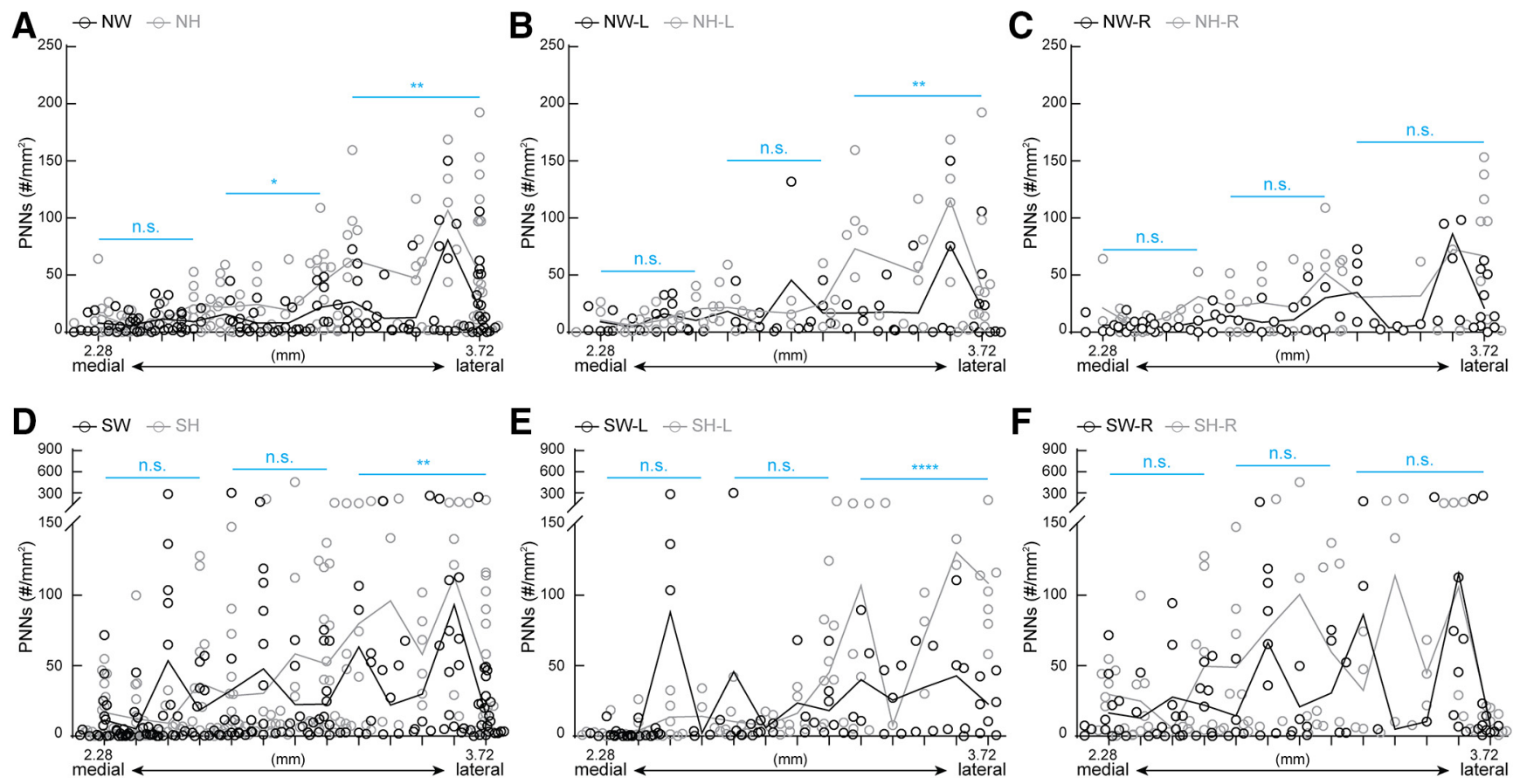

Figure 7. Left hemispheres of naïve and surrogate Mecp2 $2^{\text {Het }}$ have higher PNN density in the most lateral sections, compared to the wildtype counterparts. (A) Combined hemispheres of $\mathrm{NH}$ had significantly higher PNN density than NW, in the middle and lateral sections. No significant difference was observed in the most medial sections (medial: $n=40$ images for NW, 42 images for NH; middle: $n=32$ images for NW, 37 images for NH; lateral: $n=52$ images for NW, 44 images for NH). (B) Comparing left hemisphere PNN density between NW and $\mathrm{NH}, \mathrm{NH}$ had significantly higher PNN density in the most lateral sections, with no significant differences in the more medial sections (medial: $n=18$ images for NW-L, 23 images for NH-L; middle: $n=15$ images for NW-L, 17 images for NHL; lateral: $n=26$ images for NW-L, 29 images for NH-L). (C) There were no significant differences in the right hemisphere between $\mathrm{NW}$ and $\mathrm{NH}$, suggesting the PNN expression in the subregions of the lateral sections of the left hemisphere are particularly dysregulated in NH (medial: $n=22$ images for NW-R, 19 images for NH-R; middle: $n=18$ images for NW-R, 20 images for NH-R; lateral: $n$ $=26$ images for NW-R, 15 images for NH-R). (D) SH exhibited higher PNN density over SW only in the most lateral regions (medial: $n=62$ images for SW, 59 images for SH; middle: $n=46$ images for SW, 55 images for SH; lateral: $n=56$ images for SW, 48 images for $\mathrm{SH}$ ). (E) Similar to naïve conditions, only the lateral sections in left hemisphere of SH had significantly higher PNN density than the same regions of SW (medial: $n=28$ images for SW-L, 29 images for SH-L; middle: $n=24$ images for SW-L, 31 images for SH-L; lateral: $n=30$ images for SW-L, 20 images for SH-L). $(\boldsymbol{F})$ No significant differences between SW and SH were found in the right hemisphere (medial: $n=34$ images for SW-R, 30 images for SH-R; middle: $n=22$ images for SW-R, 24 images for SH-R; lateral: $n=$ 26 images for SW-R, 28 images for SH-R). For A-F, lines represent the mean values. Each dot represents PNN density in an individual section. Mann-Whitney test, ${ }^{*} p<0.05,{ }^{* *} p<0.01$, ${ }^{\star * \star *} p<0.0001$, n.s. = not significant. 5 mice per condition. For $\boldsymbol{A}$ and $\boldsymbol{D}, n=$ 5-24 images per map coordinate. For $\boldsymbol{B}-\boldsymbol{C}$ and $\boldsymbol{E}-\boldsymbol{F}, n=1-13$ images per map coordinate.

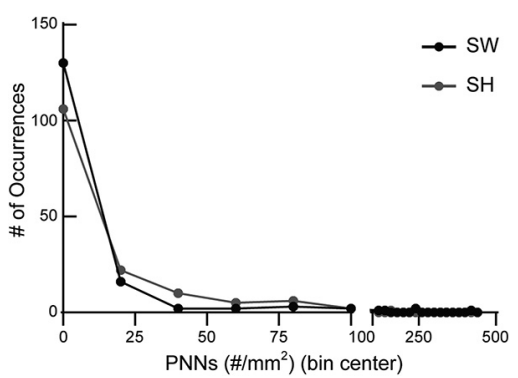

Figure 8. Significant difference in S1 PNN density between SH and SW in Table 1 due to differential distribution. Histogram analysis showed that SW had more occurrences of 0 PNN density compared to $\mathrm{SH}$, as shown by black dot (SW) above grey dot (SH). $\mathrm{SH}$ had more occurrences of 20-80 PNN density than SW, as shown by grey dots $(\mathrm{SH})$ above black dots $(\mathrm{SW})$. The average PNN density values of SW ( $n=160$ images) and SH ( $n=152$ images) are similar (Table 1) (5 mice per condition), but they are statistically different due to this differential distribution of data. distinctions in the previous figures. Overall, we observe that unsupervised analysis identifies these lateral/medial and left/right asymmetries as the major pattern that characterizes the differences in PNN distribution between experience and genotype.

\section{Individual mice exhibit strong laterality for PNN expression}

As the previous data were an aggregate/average of five biological replicates, we were interested in determining whether hemispheric biases in PNN density were seen in individual mice. For each mouse, we normalized PNN density of left hemisphere to the right hemisphere (Fig. 10). In SS1 as a whole, a modest left hemisphere bias was seen in three out of five mice across conditions and genotypes (Fig. 10A). Higher differences in left hemisphere bias is seen in subregions such as S1BF (Fig. 10B) and S1ULp (Fig. 10C). Interestingly, a decrease in the left 

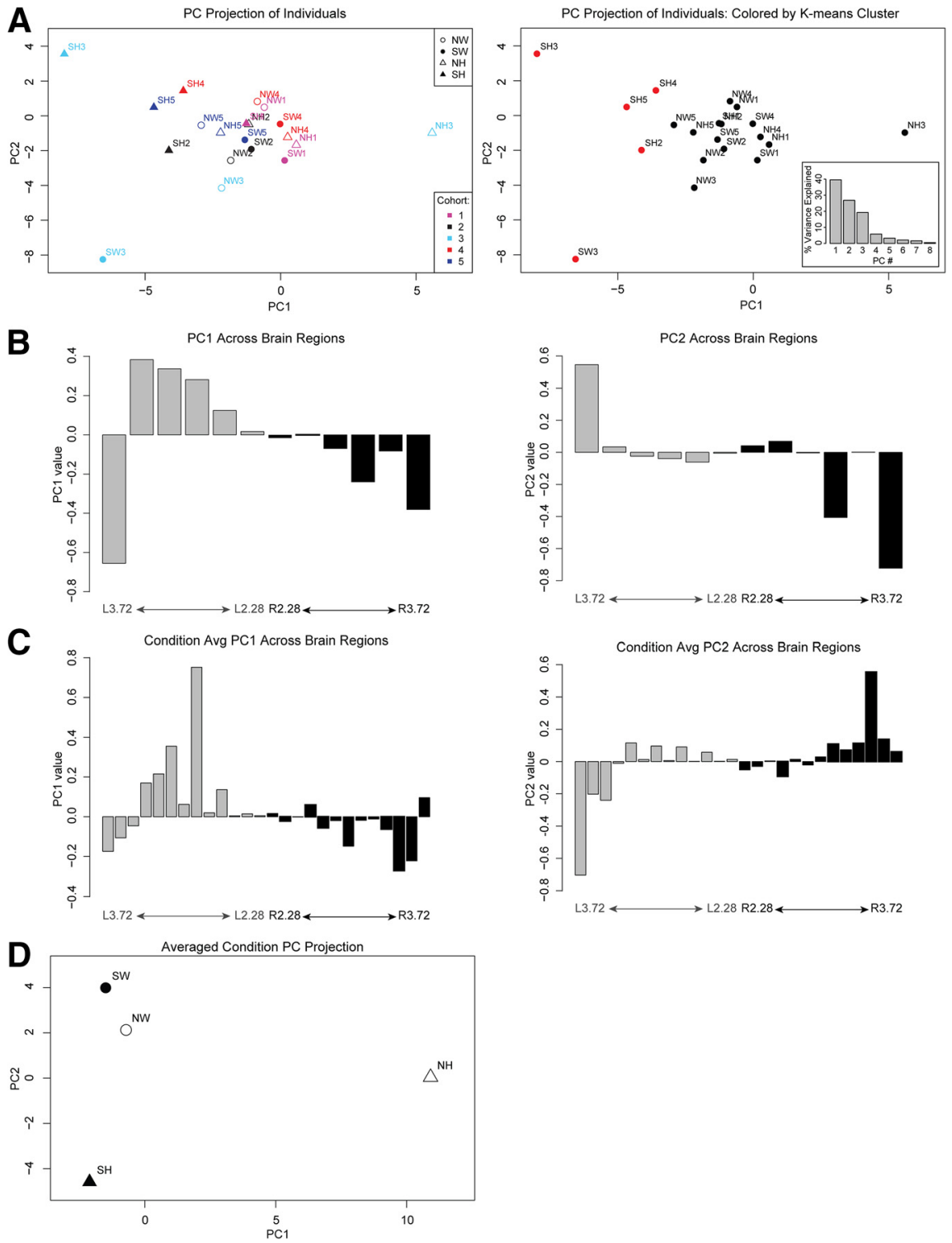

Figure 9. Principal component analysis of PNN expression segregates by conditions, lateral/medial axis and hemispheres. $(\boldsymbol{A})$ The projection of each individual brain onto PC1 and 2. These PCs are defined by relative weights of the different brain regions - all of the weights for PC1 and 2 are shown in $\boldsymbol{B}$. (Left) Individuals are colored by cohort with symbol shapes corresponding to their genotype and experience condition. (Right) Individuals are colored by K-means clustering assignment, showing that the primary separation is SH from the rest of the conditions. While PC1 tends to distinguish surrogates from naïve (in particular, surrogate het from all others), the PCA itself is an unbiased analysis that is not designed to calculate the differences in "NH vs. SH". Rather, the PC1 represents the combination of factors that are the largest source of variation among all the samples. The PC1 itself is fully defined by the weights of the different brain sections. Inset shows the \% variance explained by the different PCs, with PC1 explaining the most variance. $(\boldsymbol{B})$ Weights for each brain region for principal component (PC) 1 (left panel) and 2 (right panel) from the analysis in $(\boldsymbol{A})$. The map regions (corresponding lateral coordinates) with strongest positive and negative values contribute most strongly to the variation between individuals. The weights show a trend from medial to lateral, showing the differences in laterality are the largest source of variation in the data. (C) As in $\boldsymbol{B}$, weights for each brain region for PC1 (left panel) and 2 (right panel) are shown, in this case for PCA on data in which all cohorts were averaged for each condition. (D) Conditions projected onto PC1 show a separation of $\mathrm{NH}$ from the rest while PC2 axis shows a separation of SH from SW.

hemisphere bias was observed in most of the $\mathrm{SH}$ mice (Fig. 10A-C), suggesting that SH brains have intact plasticity mechanisms that can be triggered by this social maternal experience to overcome the abnormal high PNN density lateralization in $\mathrm{NH}$. Together, these results show that individual mice have differing hemispheric bias in
PNN density in SS1, which may contribute, in a MECP2dependent manner, to individual variability in responding to and consolidating new tasks involved in tactile sensation.

Mecp2 is a X-linked gene. It is possible that skewed MECP2 expression could lead to the individual variability 

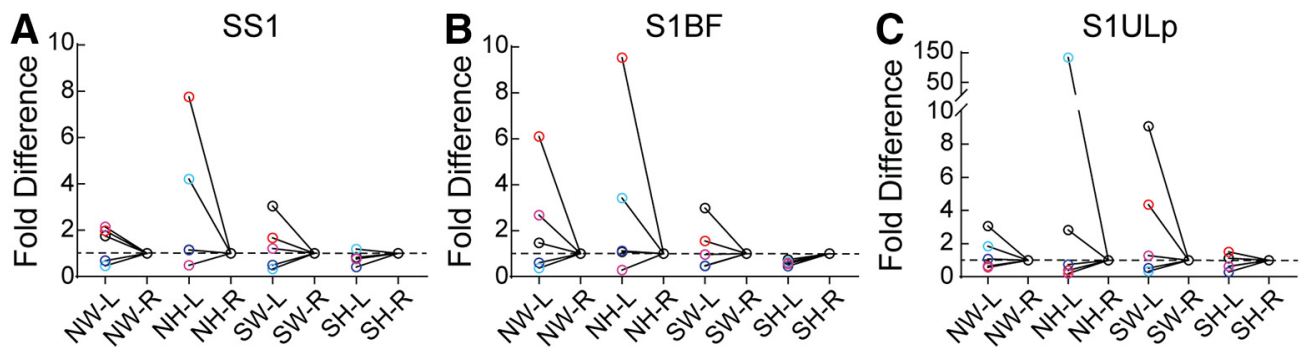

Figure 10. Individual brains exhibit differential hemispheric bias in PNN density in specific subregions of SS1. (A-C) For each brain, PNN density from the left hemisphere was normalized to the right hemisphere. This left-right hemisphere normalization revealed varying patterns of cortical asymmetry. (A) In SS1, three NW brains exhibit left hemisphere bias of 2-fold. In SW, left hemisphere bias is seen in 2 brains (black, red). Two NH brains exhibit large fold differences favoring left hemisphere (red, blue circles). In SH, no brains display cortical asymmetry. Similar trends with larger fold differences are seen in S1BF (B) and S1ULp (C).

seen in the $\mathrm{NH}$ and $\mathrm{SH}$, though this explanation does not apply to the variability seen in the WT as well. Initial observation of individual sections for all ten $\mathrm{NH}$ and $\mathrm{SH}$ brains did not show patchy, but salt-and-pepper expression of MECP2, in agreement with other work (Metcalf et al., 2006; Rietveld et al., 2015; for review, see Ribeiro and MacDonald, 2020), especially in the age of our mice used in the study (7-12 weeks old). However, we did find leftright asymmetry in MECP2 expression in both $\mathrm{NH}$ (Fig. $\left.11 A^{\prime \prime}, B^{\prime \prime}, D^{\prime \prime}, E^{\prime \prime}\right)$ and $\mathrm{SH}$ (Fig. 12A', $\left.B^{\prime \prime}, C^{\prime \prime}, E^{\prime \prime}\right)$ brains. We also found left-right asymmetry in MECP2 expression in both NW (Fig. 13, to varying degrees in most animals) and SW (Fig. 14A"', $\left.B^{\prime \prime}, E^{\prime \prime}\right)$. Together, these results suggest variable MECP2 expression between hemispheres in the same brain of either genotype (WT, Het) or condition (Naive, Sur).

\section{Discussion}

Given the long-standing and revitalized interest in extracellular matrix structures in the brain, we sought to systematically characterize high-intensity PNN expression in the whole SS1 in a model of adult experience-dependent plasticity, in relevant social behavioral conditions. To our knowledge, this is the first systematic characterization of PNN expression in the adult SS1 with detailed information about subregions and laterality in individual mice.

In early postnatal cortical development, expression of PNNs increases progressively with the maturation of that network. An excellent example is the primary visual cortex where the developmental increase of PNNs is regulated by visual experience (Beurdeley et al., 2012; Hou et al., 2017). PNNs in mature primary visual cortex mainly surround the soma and proximal dendrites of $\mathrm{PV}+$ interneurons (Hartig et al., 1992; Celio, 1993; Ueno et al., 2018). Mature PNNs are thought to be inhibitory for experiencedependent plasticity, as their increase in developing primary visual cortex correlates with the termination of the critical period and PNN removal in adult primary visual cortex restores plasticity, as measured by ocular dominance plasticity assays (Pizzorusso et al., 2002, 2006; Bavelier et al., 2010). These and studies in other brain regions (amygdala, hippocampus, piriform, and auditory cortex) have suggested that PNNs are stable, long-term structures (Sorg et al., 2016; Miyata and Kitagawa, 2017;
Ueno et al., 2019). Experiments involving the enzymatic removal of PNN by chondroitinase $\mathrm{ABC}(\mathrm{Ch} A \mathrm{BC})$ ) or hyaluronidase injections in amygdala, hippocampus, piriform and auditory cortices have shown that synaptic plasticity can be reactivated (Pizzorusso et al., 2002; Gogolla et al., 2009; Kochlamazashvili et al., 2010; Banerjee et al., 2017; Krishnan et al., 2017; Thompson et al., 2018).

A word of caution: due to ease of immunostaining with WFA and manipulation experiments with ChABC, many studies now employ PNNs as markers for plasticity. Our characterization in adult brains shows that these structures are dynamic and have hemisphere-specific and subregion-specific expression, hinting at potential neural circuitry mechanisms involving laterality in mice. Systematic and careful analysis must be taken to fully characterize PNN expression in experimental design rather than using standard "representative" sample approaches in immunostaining.

\section{What governs PNN dynamics in adults?}

Matrix metallopeptides and proteases are known to assist in remodeling extracellular matrix structures (Lu et al., 2011; Miyata and Kitagawa, 2017; Bozzelli et al., 2018). However, the contexts and mechanisms for inducing remodeling in adult brains are currently unclear. Some regional and temporal changes in PNN density have been described before (Ueno et al., 2018, 2019). However, systematic, finer scale whole-brain analysis of WFA expression across entire brain regions during development and adulthood has not been performed. Our study suggests PNNs, as measured by WFA immunostaining, may not be stable and static structures as once thought. In this study, we show that high-intensity PNNs of SS1 exhibit increased and decreased expression in a subregion-specific, hemisphere-specific manner, after maternal behavior experience. Currently, these differences in PNN expression between naive and Sur occur over one to two weeks (3-5 d before pups are born plus $6 \mathrm{~d}$ of behavior before mice are perfused). The rate of PNN formation and remodeling, which might ultimately affect tactile perception and efficient pup retrieval, remains unknown.

In the Paxinos and Franklin atlas, anatomic subregions were classified based on structural connectivity studies. Based on these anatomic characterizations, we speculate 
A NH: Left Hemisphere A' NH: Right Hemisphere A"
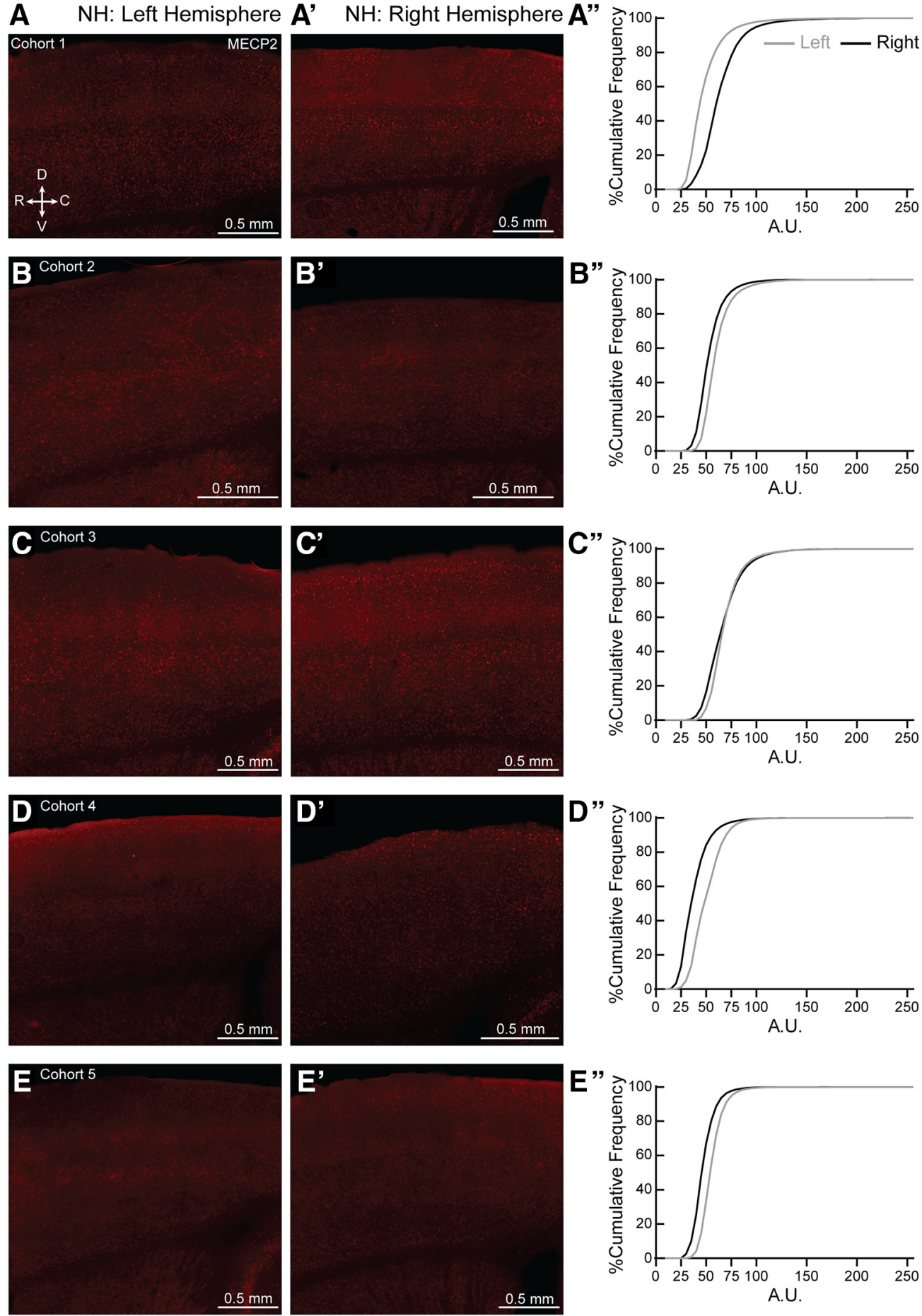

Figure 11. Hemispheric bias for MECP2 expression in individual $\mathrm{NH}$ brains. $\left(\boldsymbol{A}-\boldsymbol{E}, \boldsymbol{A}^{\prime}-\boldsymbol{E}^{\prime}\right)$ Representative $20 \mathrm{X}$ magnification, tiled projection epifluorescent images showing MECP2 expression in left $(\boldsymbol{A}-\boldsymbol{E})$ and right $\left(\boldsymbol{A}^{\prime}-\boldsymbol{E}^{\prime}\right) \mathrm{SS} 1$ of $\mathrm{NH}$ from cohorts 1-5. R $=$ rostral, $\mathrm{C}=$ caudal, $\mathrm{D}=$ dorsal and $\mathrm{V}=$ ventral. $\left(\boldsymbol{A}^{\prime \prime}-\boldsymbol{E}^{\prime \prime}\right)$ Percentage cumulative frequency distribution of MECP2 intensity within left (grey) and right (black) SS1 of the corresponding NH cohorts. Cohort $1\left(\boldsymbol{A}^{\prime \prime}\right)$ expressed more MECP2 in the right hemisphere than the left. Cohorts 2, 4-5 (B' $\boldsymbol{B}^{\prime \prime}, \boldsymbol{D}^{\prime \prime}-\boldsymbol{E}^{\prime \prime}$, respectively) expressed more MECP2 in the left hemisphere than the right, while cohort $3\left(\boldsymbol{C}^{\prime \prime}\right)$ showed similar MECP2 expression in both hemispheres. A.U. = arbitrary intensity unit. $\mathrm{N}=1$ image per hemisphere.

that changes in PNN density in specific subregions could impact information processing. For example, when NW female mice learn maternal behavior to become Sur, there is higher PNN density in the right S1BF, with a concomitant lower PNN density in left S1FL (Fig. 15, colors within IV), suggesting that these changes contribute to solidifying new synaptic contacts in S1BF, while promoting remodeling in S1FL. Together, these changes 
A SH: Left Hemisphere A' SH: Right Hemisphere A"'
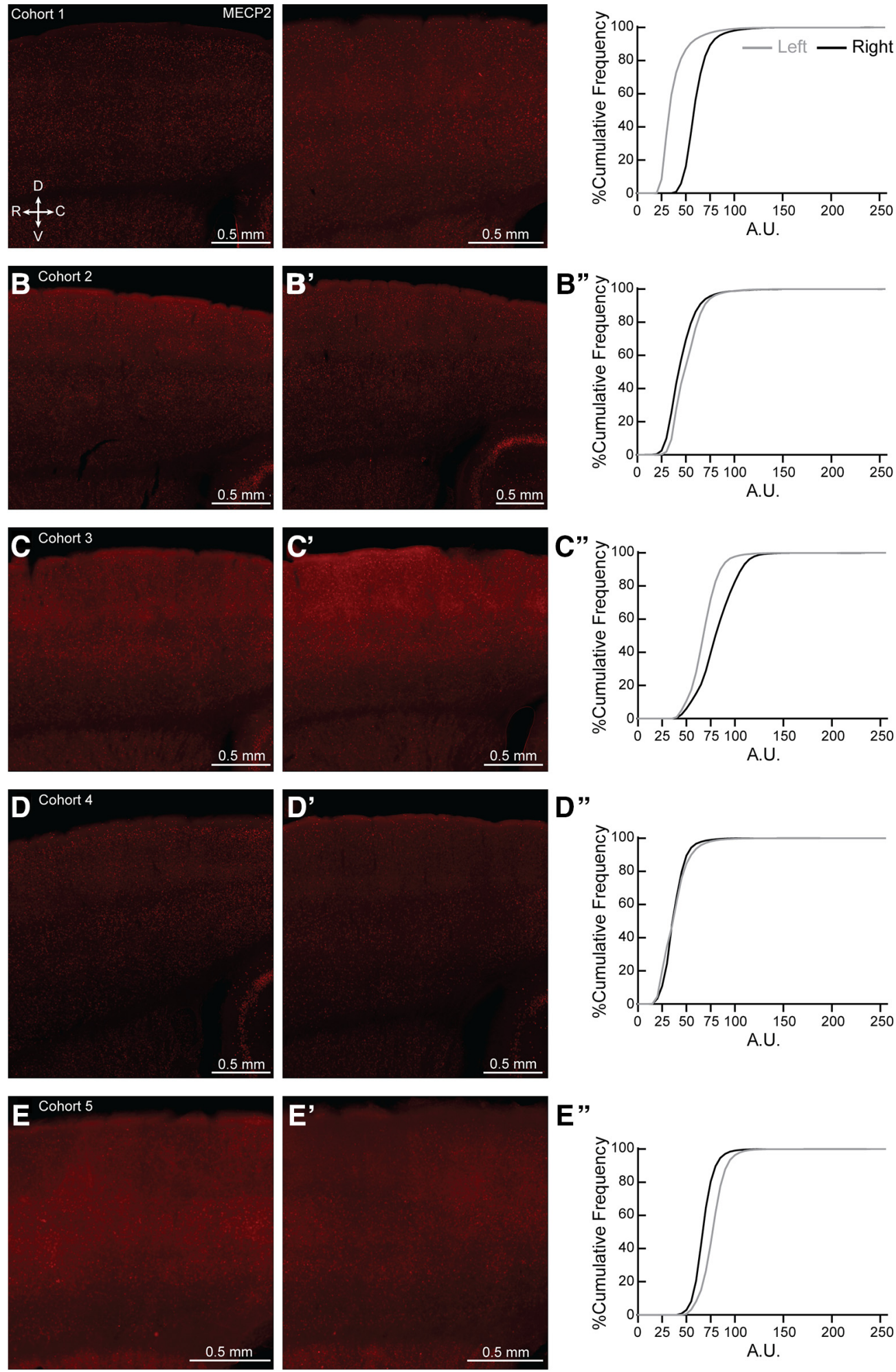

Figure 12. Hemispheric bias for MECP2 expression in individual $\mathrm{SH}$ brains. (A-E, $\left.\boldsymbol{A}^{\prime}-\boldsymbol{E}^{\prime}\right)$ Representative $20 \mathrm{X}$ magnification, tiled projection epifluorescent images showing MECP2 expression in left $(\boldsymbol{A}-\boldsymbol{E})$ and right $\left(\boldsymbol{A}^{\prime}-\boldsymbol{E}^{\prime}\right) \mathrm{SS} 1$ of $\mathrm{SH}$ from cohorts 1-5. R $=$ rostral, $\mathrm{C}=$ caudal, $\mathrm{D}=$ dorsal and $\mathrm{V}=$ ventral. $\left(\boldsymbol{A}^{\prime \prime}-\boldsymbol{E}^{\prime \prime}\right)$ Percentage cumulative frequency distribution of MECP2 intensity within left (grey) and right (black) SS1 of the corresponding SH cohorts. Cohort 1 and 3 ( $\boldsymbol{A}^{\prime \prime}$ and $\boldsymbol{C}^{\prime \prime}$, respectively) expressed more MECP2 in the right hemisphere than the left. Cohorts 2 and $5\left(\boldsymbol{B}^{\prime \prime}\right.$ and $\boldsymbol{E}^{\prime \prime}$, respectively) expressed slightly more MECP2 in the left hemisphere than the right, while cohort $4\left(\boldsymbol{D}^{\prime \prime}\right)$ showed similar MECP2 expression in both hemispheres. A.U. = arbitrary intensity unit. $\mathrm{N}=1$ image per hemisphere. 
A
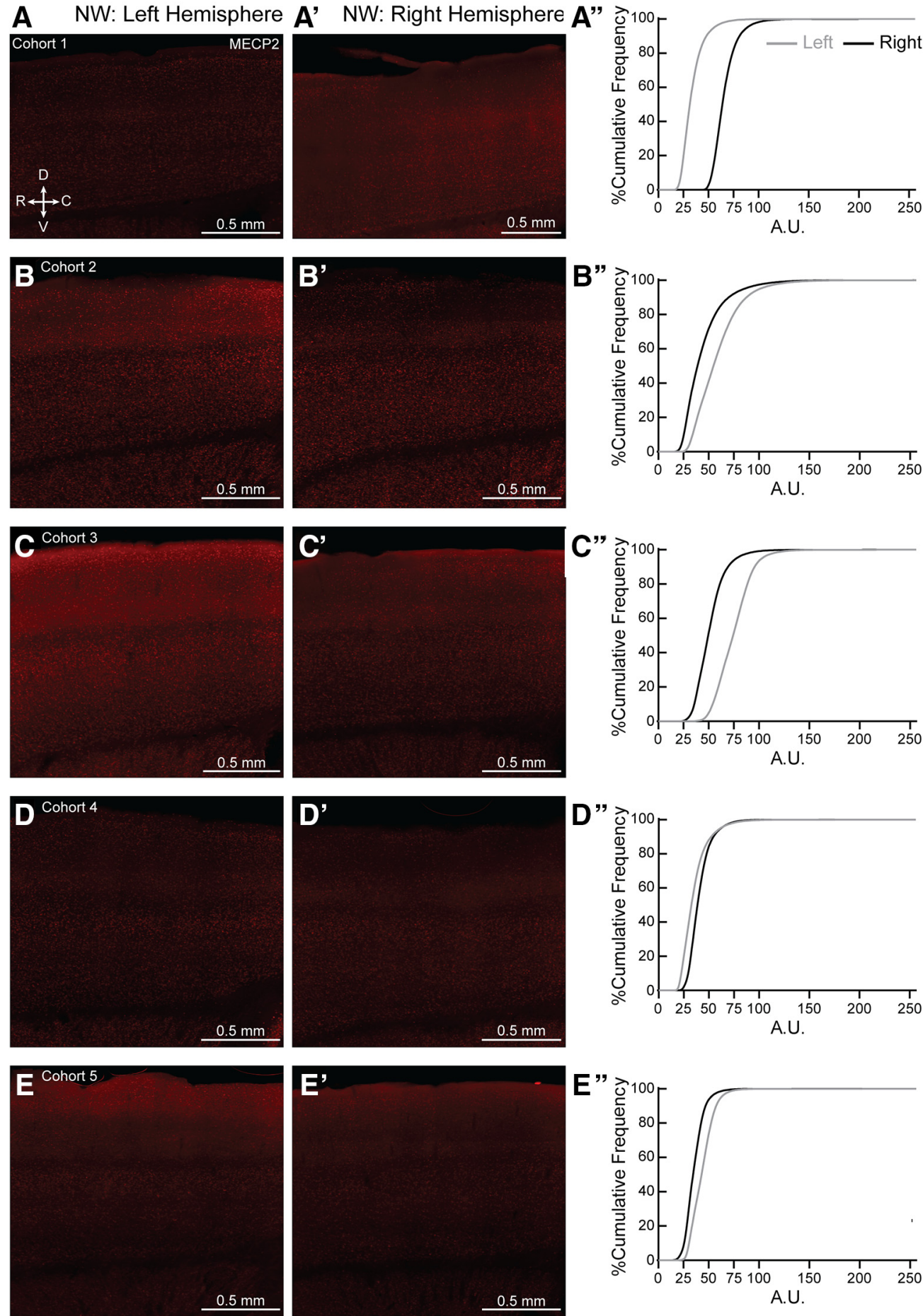

Figure 13. Hemispheric bias for MECP2 expression in individual NW brains. (A-E, $\left.\boldsymbol{A}^{\prime}-\boldsymbol{E}^{\prime}\right)$ Representative $20 \mathrm{X}$ magnification, tiled projection epifluorescent images showing MECP2 expression in left $(\boldsymbol{A}-\boldsymbol{E})$ and right $\left(\boldsymbol{A}^{\prime}-\boldsymbol{E}^{\prime}\right) \mathrm{SS} 1$ of NW from cohorts 1-5. R $=$ rostral, $\mathrm{C}=$ caudal, $\mathrm{D}=$ dorsal and $\mathrm{V}=$ ventral. $\left(\boldsymbol{A}^{\prime \prime}-\boldsymbol{E}^{\prime \prime}\right)$ Percentage cumulative frequency distribution of MECP2 intensity within left (grey) and right (black) SS1 of the corresponding NW cohorts. Cohort 1 and 4 ( $\boldsymbol{A}^{\prime \prime}$ and $\boldsymbol{D}^{\prime \prime}$, respectively) expressed more MECP2 in the right hemisphere than the left. Cohorts 2,3 and $5\left(\boldsymbol{B}^{\prime \prime}, \boldsymbol{C}^{\prime \prime}\right.$ and $\boldsymbol{E}^{\prime \prime}$, respectively) expressed more MECP2 in the left hemisphere than the right. A.U. = arbitrary intensity unit. $\mathrm{N}=1$ image per hemisphere.

could ultimately help process new tactile information related to pups and the mother acquired by the whiskers and forelimbs. Furthermore, right hemisphere specific PNN increases in S1FL and S1DZ suggests specific rewiring in subregions that could contribute to efficient information processing associated with laterality and dominant hemispheres (Fig. 15, arrows within IV). The observed fine-scale changes in PNN expression in the 
A SW: Left Hemisphere A' SW: Right Hemisphere A"'
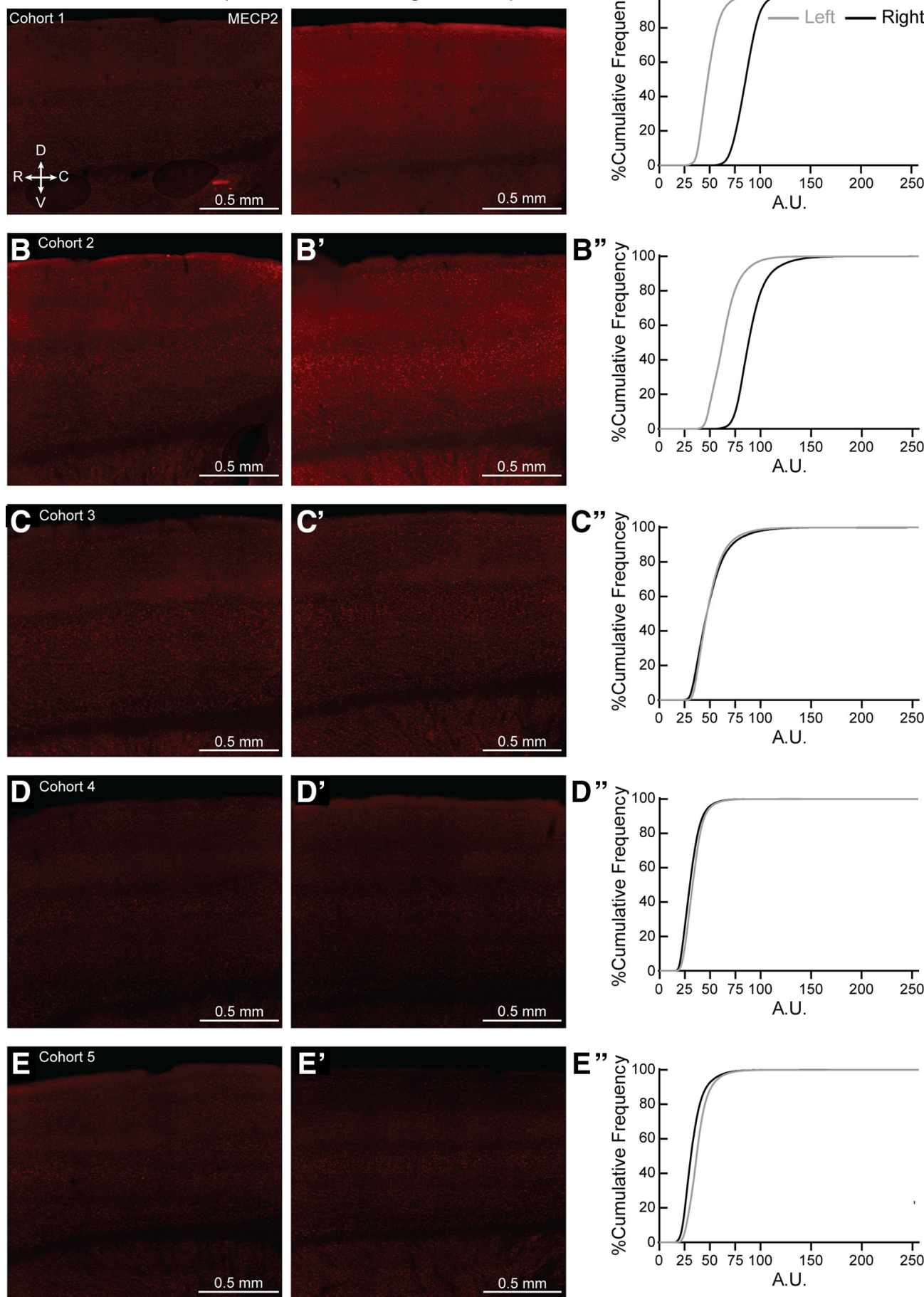

Figure 14. Hemispheric bias for MECP2 expression in individual SW brains. (A-E, $\left.\boldsymbol{A}^{\prime}-\boldsymbol{E}^{\prime}\right)$ Representative $20 \mathrm{X}$ magnification, tiled projection epifluorescent images showing MECP2 expression in left $(\boldsymbol{A}-\boldsymbol{E})$ and right $\left(\boldsymbol{A}^{\prime}-\boldsymbol{E}^{\prime}\right) \mathrm{SS} 1$ of $\mathrm{SW}$ from cohorts $1-5$. R $=$ rostral, $\mathrm{C}=$ caudal, $\mathrm{D}=$ dorsal and $\mathrm{V}=$ ventral. $\left(\boldsymbol{A}^{\prime \prime}-\boldsymbol{E}^{\prime \prime}\right)$ Percentage cumulative frequency distribution of MECP2 intensity within left (grey) and right (black) SS1 of the corresponding SW cohorts. Cohort 1 and 2 ( $\boldsymbol{A}^{\prime \prime}$ and $\boldsymbol{B}^{\prime \prime}$, respectively) expressed more MECP2 in the right hemisphere than the left. MECP2 expressions in Cohorts 3-5 $\left(\boldsymbol{C}^{\prime \prime}-\boldsymbol{E}^{\prime \prime}\right)$ were similar between left and right hemispheres. A.U. = arbitrary intensity unit. $\mathrm{N}=1$ image per hemisphere.

adult SS1 before and after maternal behavior experience suggest specific hypotheses about connectivity and functional changes in subregions, specifically in barrel field and upper lip subregions of both hemispheres. In vivo electrophysiological and/or imaging studies, which measure dynamics of neural circuitry activation and processing in intact brains, would help prove these hypotheses. 


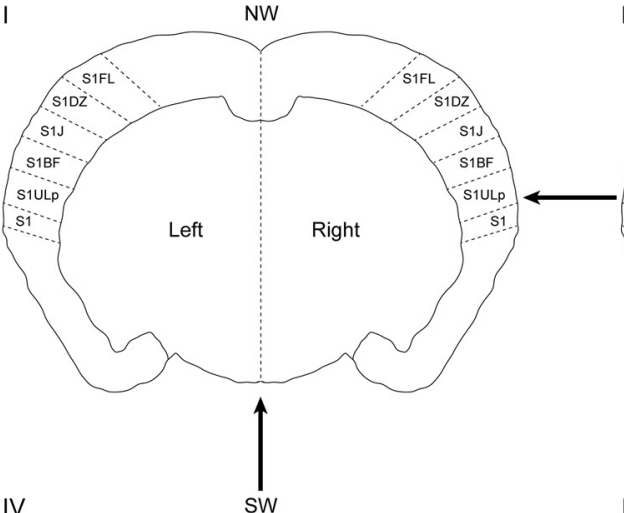

IV

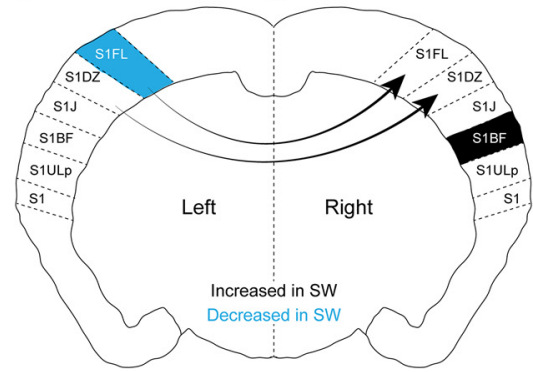

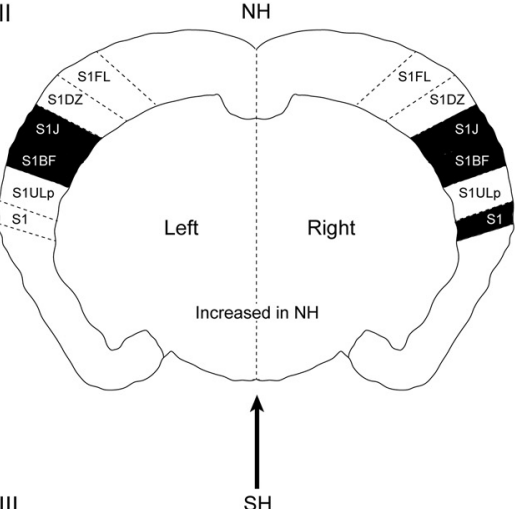

III

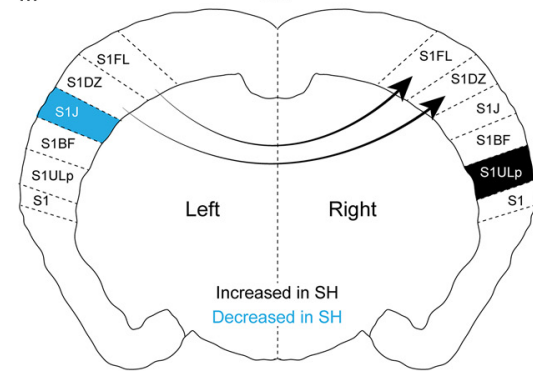

Figure 15. Summary of changes in PNN density between genotypes in maternal behavior context. (Quadrants I-IV) The changes in PNN density (grey and black shading) marked inside the brain slices denote comparisons between conditions connected by outside arrows between brain schemas. Arrows inside the brain schemas indicate hemispheric differences within genotype, with arrowheads pointing to the hemisphere with the higher PNN density. (IVàl) Comparing SW to NW, PNN density is increased in right S1BF (black) and decreased in left S1FL (blue) regions of SW. Within SW, PNN density is higher in the right hemisphere, particularly in S1FL and S1DZ. Taken together, PNN density changes in these particular subregions could contribute to tactile perception in SW, ultimately leading to efficient pup retrieval. (Ilàl) $\mathrm{NH}$ has increased PNN density in specific subregions compared to the NW, suggesting possible tactile perception issues before maternal experience, which could contribute to Mecp2 ${ }^{\text {Het'S }}$ inefficient pup retrieval performance. (IIlàll) $\mathrm{SH}$ has increased PNN density in the right S1ULp (black) and decreased PNN density in left S1J (blue), compared to $\mathrm{NH}$, suggesting possible compensatory plasticity mechanisms after maternal experience in Mecp $2^{\text {Het }}$. SH also displays higher right hemisphere PNN density in S1FL and S1DZ than its left hemisphere, similar to SW, suggesting that right hemisphere-specific increases in PNNs in S1FL and S1DZ might be important for processing tactile information during pup retrieval task.

This idea of lateralization3 of function in the rodent brain has been a topic of longstanding yet of sporadic interest in the field (Glick and Ross, 1981; Kim et al., 2012 Lu et al., 2011; Miyata and Kitagawa, 2017; Soma et al., 2017). However, the underlying cellular mechanisms governing laterality are unknown. Our novel finding that PNNs are expressed in a lateralized manner in mouse SS1 suggests that the lateralized expression could contribute to functional specialization. Pharmacological manipulations of removing PNNs unilaterally in SS1 subregions and exploring the functional consequences via electrophysiological and behavioral studies are ongoing. Previously we showed that, in this adult female mouse model for Rett syndrome (Het), PNNs were increased in a transient atypical manner in the auditory cortex, which correlated with their inefficient pup retrieval (Krishnan et al., 2017). Manipulating auditory cortex PNNs by ChABC injections or genetic reductions in Het significantly improved aspects of $\mathrm{SH}$ pup retrieval behavior, showing that PNNs play crucial roles in learning and executing this behavior (Krishnan et al., 2017). We recently showed that aberrant PNN expression leads to dysregulation of auditory cortical PV networks in Het, while behaviorally-relevant pup vocalization stimuli were presented during in vivo awake electrophysiological recordings (Lau et al., 2020). Here, we showed that $\mathrm{NH}$ already have abnormal increased PNN expression in subregions of SS1 compared with NW (Fig. 15, II), and surrogacy further increased PNN expressions (Fig. 15, III). Current results suggest information flow, network activation and multisensory integration could be affected in Het, in specific cortical regions such as SS1 and auditory cortex (Krishnan et al., 2017; Morello et al., 2018; Lau et al., 2020). Further whole brain analysis on laterality in PNN density in the auditory cortex is warranted, especially due to reports suggesting left hemisphere-specific neural circuitry activation (Ehret et al., 1987; Stiebler et al., 1997; Marlin et al., 2015).

\section{What regulates expression of PNN proteins?}

Changes in PNN expression in the early developing cortex correlates with the increasing expression of MECP2, PV, and other components of the GABAergic machinery in $\mathrm{PV}+\mathrm{GABAergic}$ neurons (Krishnan et al., 2015). Expression of MECP2, PV (calcium binding protein), GAD67 (major enzyme that makes GABA in the cortex) and PNNs can dynamically change with activity, in both developing and adult brains (Ye and Miao, 2013; Krishnan et al., 2015, 2017). MECP2 directly occupies the promoter regions of Gad1 (gene that makes GAD67) and PV (Chao et al., 2010; Durand et al., 2012), thus potentially configuring chromatin in these promoter and enhancer regions for appropriate activity-dependent and experience-dependent regulation. MECP2 regulates many genes (Cohen et al., 2011b; Gabel et al., 2015); therefore, genes encoding for PNN proteins could also be regulated by MECP2. Previously, we showed that the expression of mature PNNs is accelerated in the developing male Mecp2-null primary visual cortex (Krishnan et al., 2015), suggesting MECP2 could act as a repressor of PNNs. However, reducing GABAergic inhibition by using a Gad1 Het allele in Mecp2-mutant backgrounds 
reduced PNN expression to WT levels (Krishnan et al., $2015,2017)$, suggesting that expression of the extracellular matrix proteins that ultimately form PNNs may not require direct control of MECP2 and is amenable to change with dynamic inhibition changes.

\section{Mosaicism in MECP2 expression and Het variability}

Most previous studies in mouse models of RTT were conducted in Mecp2-null male mice, because they exhibit earlier and more severe phenotypes in many standard assays. Therefore, with the exception of a few studies (Stearns et al., 2007; Garg et al., 2013; Samaco et al., 2013; Ribeiro and MacDonald, 2020), the molecular, circuit and behavioral defects in Mecp2 ${ }^{\text {Het }}$ female mice are largely unknown. Since RTT affects predominantly females, $M e c p 2^{\text {Het }}$ female mice represent a more translationally relevant model of RTT than Mecp2-null male mice, though they are rarely used in studies due to random $\mathrm{X}$ chromosome inactivation patterns contributing to variability in phenotypes. In the alloparental behavioral model, we have shown that even with mosaic expression of MECP2 in the auditory cortex of Het, higher MECP2 expression correlates well with better efficiency during pup gathering behavior (Krishnan et al., 2017). MECP2 is known to have different levels in different cell types (Lioy et al., 2011; Lee et al., 2014; Song et al., 2014; Rakela et al., 2018). MECP2 protein function can be regulated by activity-dependent post-translational mechanisms (Cohen et al., 2011b; Ebert et al., 2013; Gabel et al., 2015); however, these studies do not take laterality or regional differences into account. Here, we find that MECP2 expression is asymmetric between left and right hemispheres of SS1, in both WT and Het with individual variations across brains (Figs. 11-14). These results suggest a more in-depth and systematic analysis is required to determine how variable MECP2 expression in hemispheres, subregions and cell types over time could result in diverse phenotypes. Maternal or paternal allele activation in individual cells should also be taken into consideration, as it could contribute to laterality in CNS cell types (Wu et al., 2014).

\section{References}

Amir RE, Van den Veyver IB, Wan M, Tran CQ, Francke U, Zoghbi HY (1999) Rett syndrome is caused by mutations in X-linked MECP2, encoding methyl-CpG-binding protein 2. Nat Genet 23:185-188.

Banerjee SB, Gutzeit VA, Baman J, Aoued HS, Doshi NK, Liu RC, Ressler KJ (2017) Perineuronal nets in the adult sensory cortex are necessary for fear learning. Neuron 95:169-179.e3.

Bartos M, Vida I, Frotscher M, Meyer A, Monyer H, Geiger JRP, Jonas P (2002) Fast synaptic inhibition promotes synchronized gamma oscillations in hippocampal interneuron networks. Proc Natl Acad Sci USA 99:13222-13227.

Bavelier D, Levi DM, Li RW, Dan Y, Hensch TK (2010) Removing brakes on adult brain plasticity: from molecular to behavioral interventions. J Neurosci 30:14964-14971.

Beach FA, Jaynes J (1956) Studies of maternal retrieving in rats. III. Sensory cues involved in the lactating female's response to her young. Behaviour 10:104-124.

Becker A, Zhang P, Allmann L, Meilinger D, Bertulat B, Eck D, Hofstaetter M, Bartolomei G, Hottiger MO, Schreiber V, Leonhardt H, Cardoso MC (2016) Poly(ADP-ribosyl)ation of methyl CpG binding domain protein 2 regulates chromatin structure. $\mathrm{J}$ Biol Chem 291:4873-4881.

Begum MR, Sng JCG (2017) Molecular mechanisms of experiencedependent maturation in cortical GABAergic inhibition. J Neurochem 142:649-661.

Bernard C, Prochiantz A (2016) Otx2-PNN interaction to regulate cortical plasticity. Neural Plast 2016:7931693.

Berretta S, Pantazopoulos H, Markota M, Brown C, Batzianouli ET (2015) Losing the sugar coating: potential impact of perineuronal net abnormalities on interneurons in schizophrenia. Schizophr Res 167:18-27.

Beurdeley M, Spatazza J, Lee HH, Sugiyama S, Bernard C, Di Nardo AA, Hensch TK, Prochiantz A (2012) Otx2 binding to perineuronal nets persistently regulates plasticity in the mature visual cortex. $J$ Neurosci 32:9429-9437.

Bignami A, Asher R, Perides G (1992) Co-localization of hyaluronic acid and chondroitin sulfate proteoglycan in rat cerebral cortex. Brain Res 579:173-177.

Bozzelli PL, Alaiyed S, Kim E, Villapol S, Conant K (2018) Proteolytic remodeling of perineuronal nets: effects on synaptic plasticity and neuronal population dynamics. Neural Plast 2018:5735789.

Brecht M (2007) Barrel cortex and whisker-mediated behaviors. Curr Opin Neurobiol 17:408-416.

Brückner G, Bringmann A, Köppe G, Härtig W, Brauer K (1996) In vivo and in vitro labelling of perineuronal nets in rat brain. Brain Res 720:84-92.

Brückner G, Morawski M, Arendt T (2008) Aggrecan-based extracellular matrix is an integral part of the human basal ganglia circuit. Neuroscience 151:489-504.

Cabungcal $J H$, Steullet $P$, Morishita $H$, Kraftsik R, Cuenod $M$, Hensch TK, Do KQ (2013) Perineuronal nets protect fast-spiking interneurons against oxidative stress. Proc Natl Acad Sci USA 110:9130-9135.

Carstens KE, Phillips ML, Pozzo-Miller L, Weinberg RJ, Dudek SM (2016) Perineuronal nets suppress plasticity of excitatory synapses on CA2 pyramidal neurons. J Neurosci 36:6312-6320.

Carulli D, Rhodes KE, Fawcett JW (2007) Upregulation of aggrecan, link protein 1, and hyaluronan synthases during formation of perineuronal nets in the rat cerebellum. J Comp Neurol 501:83-94.

Cattaud V, Bezzina C, Rey CC, Lejards C, Dahan L, Verret L (2018) Early disruption of parvalbumin expression and perineuronal nets in the hippocampus of the Tg2576 mouse model of Alzheimer's disease can be rescued by enriched environment. Neurobiol Aging 72:147-158.

Celio MR (1993) Perineuronal nets of extracellular matrix around parvalbumin-containing neurons of the hippocampus. Hippocampus 3:55-60.

Chahrour M, Jung SY, Shaw C, Zhou X, Wong STC, Qin J, Zoghbi HY (2008) MeCP2, a key contributor to neurological disease, activates and represses transcription. Science 320:1224-1229.

Chao HT, Chen H, Samaco RC, Xue M, Chahrour M, Yoo J, Neul JL, Gong S, Lu HC, Heintz N, Ekker M, Rubenstein JLR, Noebels JL, Rosenmund C, Zoghbi HY (2010) Dysfunction in GABA signalling mediates autism-like stereotypies and Rett syndrome phenotypes. Nature 468:263-269.

Chapin JK, Lin CS (1984) Mapping the body representation in the SI cortex of anesthetized and awake rats. J Comp Neurol 229:199213.

Cohen L, Rothschild G, Mizrahi A (2011a) Multisensory integration of natural odors and sounds in the auditory cortex. Neuron 72:357369.

Cohen S, Gabel HW, Hemberg M, Hutchinson AN, Sadacca LA, Ebert DH, Harmin DA, Greenberg RS, Verdine VK, Zhou Z, Wetsel WC, West AE, Greenberg ME (2011b) Genome-wide activity-dependent MeCP2 phosphorylation regulates nervous system development and function. Neuron 72:72-85.

Dani VS, Chang Q, Maffei A, Turrigiano GG, Jaenisch R, Nelson SB (2005) Reduced cortical activity due to a shift in the balance between excitation and inhibition in a mouse model of Rett syndrome. Proc Natl Acad Sci USA 102:12560-12565. 
Deepa SS, Umehara Y, Higashiyama S, Itoh N, Sugahara K (2002) Specific molecular interactions of oversulfated chondroitin sulfate $\mathrm{E}$ with various heparin-binding growth factors. Implications as a physiological binding partner in the brain and other tissues. J Biol Chem 277:43707-43716.

de Winter F, Kwok JCF, Fawcett JW, Vo TT, Carulli D, Verhaagen J (2016) The chemorepulsive protein semaphorin $3 A$ and perineuronal net-mediated plasticity. Neural Plast 2016:3679545.

Dityatev A, Brückner G, Dityateva G, Grosche J, Kleene R, Schachner M (2007) Activity-dependent formation and functions of chondroitin sulfate-rich extracellular matrix of perineuronal nets. Dev Neurobiol 67:570-588.

Donato F, Rompani SB, Caroni P (2013) Parvalbumin-expressing basket-cell network plasticity induced by experience regulates adult learning. Nature 504:272-276.

Durand S, Patrizi A, Quast KB, Hachigian L, Pavlyuk R, Saxena A, Carninci P, Hensch TK, Fagiolini M (2012) NMDA receptor regulation prevents regression of visual cortical function in the absence of Mecp2. Neuron 76:1078-1090.

Ebert DH, Gabel HW, Robinson ND, Kastan NR, Hu LS, Cohen S, Navarro AJ, Lyst MJ, Ekiert R, Bird AP, Greenberg ME (2013) Activity-dependent phosphorylation of MeCP2 threonine 308 regulates interaction with NCoR. Nature 499:341-345.

Ehret G, Koch M, Haack B, Markl H (1987) Sex and parental experience determine the onset of an instinctive behavior in mice. Naturwissenschaften 74:47.

Enwright JF, Sanapala S, Foglio A, Berry R, Fish KN, Lewis DA (2016) Reduced labeling of parvalbumin neurons and perineuronal nets in the dorsolateral prefrontal cortex of subjects with schizophrenia. Neuropsychopharmacology 41:2206-2214.

Feldman DE, Brecht M (2005) Map plasticity in somatosensory cortex. Science 310:810-815.

Frischknecht R, Heine M, Perrais D, Seidenbecher Cl, Choquet D, Gundelfinger ED (2009) Brain extracellular matrix affects AMPA receptor lateral mobility and short-term synaptic plasticity. Nat Neurosci 12:897-904.

Gabel HW, Kinde B, Stroud H, Gilbert CS, Harmin DA, Kastan NR, Hemberg M, Ebert DH, Greenberg ME (2015) Disruption of DNAmethylation-dependent long gene repression in Rett syndrome. Nature 522:89-93.

Galindo-Leon EE, Lin FG, Liu RC (2009) Inhibitory plasticity in a lateral band improves cortical detection of natural vocalizations. Neuron 62:705-716.

Garg SK, Lioy DT, Cheval H, McGann JC, Bissonnette JM, Murtha MJ, Foust KD, Kaspar BK, Bird A, Mandel G (2013) Systemic delivery of MeCP2 rescues behavioral and cellular deficits in female mouse models of Rett syndrome. J Neurosci 33:13612-13620.

Glick SD, Ross DA (1981) Lateralization of function in the rat brain. Trends Neurosci 4:196-199.

Gogolla N, Caroni P, Lüthi A, Herry C (2009) Perineuronal nets protect fear memories from erasure. Science 325:1258-1261.

Gundelfinger ED, Frischknecht R, Choquet D, Heine M (2010) Converting juvenile into adult plasticity: a role for the brain's extracellular matrix: converting juvenile into adult plasticity. Eur $\mathrm{J}$ Neurosci 31:2156-2165.

Guy J, Hendrich B, Holmes M, Martin JE, Bird A (2001) A mouse Mecp2-null mutation causes neurological symptoms that mimic Rett syndrome. Nat Genet 27:322-326.

Hartig W, Brauer K, Bruckner G (1992) Wisteria floribunda agglutinin-labelled nets surround parvalbumin-containing neurons. Neuroreport 3:869-872.

Hou X, Yoshioka N, Tsukano H, Sakai A, Miyata S, Watanabe Y, Yanagawa Y, Sakimura K, Takeuchi K, Kitagawa H, Hensch TK, Shibuki K, Igarashi M, Sugiyama S (2017) Chondroitin sulfate is required for onset and offset of critical period plasticity in visual cortex. Sci Rep 7.

Kalemaki K, Konstantoudaki X, Tivodar S, Sidiropoulou K, Karagogeos D (2018) Mice with decreased number of interneurons exhibit aberrant spontaneous and oscillatory activity in the cortex. Front Neural Circuits 12:96.
Kenyon P, Cronin P, Keeble S (1981) Disruption of maternal retrieving by perioral anesthesia. Physiol Behav 27:313-321.

Kim S, Mátyás F, Lee S, Acsády L, Shin HS (2012) Lateralization of observational fear learning at the cortical but not thalamic level in mice. Proc Natl Acad Sci USA 109:15497-15501.

Kochlamazashvili G, Henneberger C, Bukalo O, Dvoretskova E, Senkov O, Lievens PM-J, Westenbroek R, Engel AK, Catterall WA, Rusakov DA, Schachner M, Dityatev A (2010) The extracellular matrix molecule hyaluronic acid regulates hippocampal synaptic plasticity by modulating postsynaptic L-type $\mathrm{Ca}(2+)$ channels. Neuron 67:116-128.

Kosaka T, Heizmann CW (1989) Selective staining of a population of parvalbumin-containing GABAergic neurons in the rat cerebral cortex by lectins with specific affinity for terminal $\mathrm{N}$-acetylgalactosamine. Brain Res 483:158-163.

Krishnan K, Wang BS, Lu J, Wang L, Maffei A, Cang J, Huang ZJ (2015) MeCP2 regulates the timing of critical period plasticity that shapes functional connectivity in primary visual cortex. Proc Natl Acad Sci USA 112:E4782-E4791.

Krishnan K, Lau BYB, Ewall G, Huang ZJ, Shea SD (2017) MECP2 regulates cortical plasticity underlying a learned behaviour in adult female mice. Nat Commun 8:14077.

Kwok JCF, Carulli D, Fawcett JW (2010) In vitro modeling of perineuronal nets: hyaluronan synthase and link protein are necessary for their formation and integrity. J Neurochem 114:1447-1459.

Lagger S, Connelly JC, Schweikert G, Webb S, Selfridge J, Ramsahoye BH, Yu M, He C, Sanguinetti G, Sowers LC, Walkinshaw MD, Bird A (2017) MeCP2 recognizes cytosine methylated tri-nucleotide and di-nucleotide sequences to tune transcription in the mammalian brain. PLoS Genet 13:e1006793.

Lau BYB, Krishnan K, Huang ZJ, Shea SD (2020) Maternal experience-dependent cortical plasticity in mice is circuit- and stimulusspecific and requires MECP2. J Neurosci 40:1514-1526.

Lee T, Kim U (2012) Descending projections from the dysgranular zone of rat primary somatosensory cortex processing deep somatic input. J Comp Neurol 520:1021-1046.

Lee W, Yun JM, Woods R, Dunaway K, Yasui DH, Lasalle JM, Gong Q (2014) MeCP2 regulates activity-dependent transcriptional responses in olfactory sensory neurons. Hum Mol Genet 23:63666374.

Lioy DT, Garg SK, Monaghan CE, Raber J, Foust KD, Kaspar BK, Hirrlinger PG, Kirchhoff F, Bissonnette JM, Ballas N, Mandel G (2011) A role for glia in the progression of Rett's syndrome. Nature 475:497-500.

Lonstein JS, Stern JM (1997) Somatosensory contributions to c-fos activation within the caudal periaqueductal gray of lactating rats: effects of perioral, rooting, and suckling stimuli from pups. Horm Behav 32:155-166.

Lonstein JS, Lévy F, Fleming AS (2015) Common and divergent psychobiological mechanisms underlying maternal behaviors in nonhuman and human mammals. Horm Behav 73:156-185.

Lu P, Takai K, Weaver VM, Werb Z (2011) Extracellular matrix degradation and remodeling in development and disease. Cold Spring Harb Perspect Biol 3.

Marlin BJ, Mitre M, D'amour JA, Chao MV, Froemke RC (2015) Oxytocin enables maternal behaviour by balancing cortical inhibition. Nature 520:499-504.

Metcalf BM, Mullaney BC, Johnston MV, Blue ME (2006) Temporal shift in methyl-CpG binding protein 2 expression in a mouse model of Rett syndrome. Neuroscience 139:1449-1460.

Miyata S, Kitagawa H (2017) Formation and remodeling of the brain extracellular matrix in neural plasticity: roles of chondroitin sulfate and hyaluronan. Biochim Biophys Acta Gen Subj 1861:24202434.

Miyata S, Komatsu Y, Yoshimura Y, Taya C, Kitagawa H (2012) Persistent cortical plasticity by upregulation of chondroitin 6-sulfation. Nat Neurosci 15:414-422.

Morawski M, Brückner MK, Riederer P, Brückner G, Arendt T (2004) Perineuronal nets potentially protect against oxidative stress. Exp Neurol 188:309-315. 
Morello N, Schina R, Pilotto F, Phillips M, Melani R, Plicato O, Pizzorusso T, Pozzo-Miller L, Giustetto M (2018) Loss of Mecp2 causes atypical synaptic and molecular plasticity of parvalbuminexpressing interneurons reflecting Rett syndrome-like sensorimotor defects. eNeuro 5.

Morgan HD, Fleming AS, Stern JM (1992) Somatosensory control of the onset and retention of maternal responsiveness in primiparous Sprague-Dawley rats. Physiol Behav 51:549-555.

Muotri AR, Marchetto MCN, Coufal NG, Oefner R, Yeo G, Nakashima K, Gage FH (2010) L1 retrotransposition in neurons is modulated by MeCP2. Nature 468:443-446.

Murthy S, Kane GA, Katchur NJ, Lara Mejia PS, Obiofuma G, Buschman TJ, McEwen BS, Gould E (2019) Perineuronal nets, inhibitory interneurons, and anxiety-related ventral hippocampal neuronal oscillations are altered by early life adversity. Biol Psychiatry 85:1011-1020.

Nakagawa F, Schulte BA, Wu JY, Spicer SS (1986) GABAergic neurons of rodent brain correspond partially with those staining for glycoconjugate with terminal $\mathrm{N}$-acetylgalactosamine. J Neurocytol 15:389-396.

Noutel J, Hong YK, Leu B, Kang E, Chen C (2011) Experience-dependent retinogeniculate synapse remodeling is abnormal in MeCP2-deficient mice. Neuron 70:35-42.

Orlando C, Ster J, Gerber U, Fawcett JW, Raineteau O (2012) Perisynaptic chondroitin sulfate proteoglycans restrict structural plasticity in an integrin-dependent manner. J Neurosci 32:18009-18017.

Pantazopoulos H, Markota M, Jaquet F, Ghosh D, Wallin A, Santos A, Caterson B, Berretta S (2015) Aggrecan and chondroitin-6-sulfate abnormalities in schizophrenia and bipolar disorder: a postmortem study on the amygdala. Transl Psychiatry 5:e496.

Paxinos G, Franklin KBJ (2013) The mouse brain in stereotaxic coordinates, Ed 4. Waltham: Academic Press.

Picard N, Fagiolini M (2019) MeCP2: an epigenetic regulator of critical periods. Curr Opin Neurobiol 59:95-101.

Pizzo R, Gurgone A, Castroflorio E, Amendola E, Gross C, SassoèPognetto M, Giustetto M (2016) Lack of Cdkl5 disrupts the organization of excitatory and inhibitory synapses and parvalbumin interneurons in the primary visual cortex. Front Cell Neurosci 10:261.

Pizzorusso T, Medini P, Berardi N, Chierzi S, Fawcett JW, Maffei L (2002) Reactivation of ocular dominance plasticity in the adult visual cortex. Science 298:1248-1251.

Pizzorusso T, Medini P, Landi S, Baldini S, Berardi N, Maffei L (2006) Structural and functional recovery from early monocular deprivation in adult rats. Proc Natl Acad Sci USA 103:8517-8522.

Rakela B, Brehm P, Mandel G (2018) Astrocytic modulation of excitatory synaptic signaling in a mouse model of Rett syndrome. Elife 7.

Reinhard SM, Rais M, Afroz S, Hanania Y, Pendi K, Espinoza K, Rosenthal R, Binder DK, Ethell IM, Razak KA (2019) Reduced perineuronal net expression in Fmr1 KO mice auditory cortex and amygdala is linked to impaired fear-associated memory. Neurobiol Learn Mem 164:107042.

Ribeiro MC, MacDonald JL (2020) Sex differences in Mecp2-mutant Rett syndrome model mice and the impact of cellular mosaicism in phenotype development. Brain Res 1729:146644.

Rietveld L, Stuss DP, McPhee D, Delaney KR (2015) Genotype-specific effects of Mecp2 loss-of-function on morphology of layer $V$ pyramidal neurons in heterozygous female Rett syndrome model mice. Front Cell Neurosci 9.

Samaco RC, McGraw CM, Ward CS, Sun Y, Neul JL, Zoghbi HY (2013) Female Mecp2+/- mice display robust behavioral deficits on two different genetic backgrounds providing a framework for pre-clinical studies. Hum Mol Genet 22:96-109.

Schneider CA, Rasband WS, Eliceiri KW (2012) NIH Image to ImageJ: 25 years of image analysis. Nat Methods 9:671-675.

Shin Yim Y, Park A, Berrios J, Lafourcade M, Pascual LM, Soares N, Yeon Kim J, Kim S, Kim H, Waisman A, Littman DR, Wickersham IR, Harnett MT, Huh JR, Choi GB (2017) Reversing behavioural abnormalities in mice exposed to maternal inflammation. Nature 549:482-487.
Sigal YM, Bae H, Bogart LJ, Hensch TK, Zhuang X (2019) Structural maturation of cortical perineuronal nets and their perforating synapses revealed by superresolution imaging. Proc Natl Acad Sci USA 116:7071-7076.

Skene PJ, Illingworth RS, Webb S, Kerr ARW, James KD, Turner DJ, Andrews R, Bird AP (2010) Neuronal MeCP2 is expressed at near histone-octamer levels and globally alters the chromatin state. Mol Cell 37:457-468.

Soma S, Saiki A, Yoshida J, Ríos A, Kawabata M, Sakai Y, Isomura Y (2017) Distinct laterality in forelimb-movement representations of rat primary and secondary motor cortical neurons with intratelencephalic and pyramidal tract projections. J Neurosci 37:10904-10916.

Song C, Feodorova Y, Guy J, Peichl L, Jost KL, Kimura H, Cardoso MC, Bird A, Leonhardt H, Joffe B, Solovei I (2014) DNA methylation reader MECP2: cell type- and differentiation stage-specific protein distribution. Epigenetics Chromatin 7:17.

Sorg BA, Berretta S, Blacktop JM, Fawcett JW, Kitagawa H, Kwok JCF, Miquel M (2016) Casting a wide net: role of perineuronal nets in neural plasticity. J Neurosci 36:11459-11468.

Stearns NA, Schaevitz LR, Bowling H, Nag N, Berger UV, BergerSweeney J (2007) Behavioral and anatomical abnormalities in Mecp2 mutant mice: a model for Rett syndrome. Neuroscience 146:907-921.

Stern JM (1996) Somatosensation and maternal care in Norway rats. In: Advances in the study of behavior, pp 243-294. San Diego: Elsevier.

Stiebler I, Neulist R, Fichtel I, Ehret G (1997) The auditory cortex of the house mouse: left-right differences, tonotopic organization and quantitative analysis of frequency representation. J Comp Physiol A Neuroethol Sens Neural Behav Physiol 181:559-571.

Stolzenberg DS, Champagne FA (2016) Hormonal and non-hormonal bases of maternal behavior: the role of experience and epigenetic mechanisms. Horm Behav 77:204-210.

Sugiyama S, Prochiantz A, Hensch TK (2009) From brain formation to plasticity: insights on Otx2 homeoprotein: Otx2 transfer shapes postnatal circuits. Dev Growth Differ 51:369-377.

Suttkus A, Rohn S, Jäger C, Arendt T, Morawski M (2012) Neuroprotection against iron-induced cell death by perineuronal nets - an in vivo analysis of oxidative stress. Am $\mathrm{J}$ Neurodegener Dis 1:122-129.

Thompson EH, Lensjø KK, Wigestrand MB, Malthe-Sørenssen A, Hafting T, Fyhn M (2018) Removal of perineuronal nets disrupts recall of a remote fear memory. Proc Natl Acad Sci USA 115:607-612.

Ueno H, Takao K, Suemitsu S, Murakami S, Kitamura N, Wani K, Okamoto M, Aoki S, Ishihara T (2018) Age-dependent and regionspecific alteration of parvalbumin neurons and perineuronal nets in the mouse cerebral cortex. Neurochem Int 112:59-70.

Ueno H, Suemitsu S, Murakami S, Kitamura N, Wani K, Matsumoto Y, Okamoto M, Ishihara T (2019) Layer-specific expression of extracellular matrix molecules in the mouse somatosensory and piriform cortices. IBRO Rep 6:1-17.

Vo T, Carulli D, Ehlert EME, Kwok JCF, Dick G, Mecollari V, Moloney EB, Neufeld G, de Winter F, Fawcett JW, Verhaagen J (2013) The chemorepulsive axon guidance protein semaphorin3A is a constituent of perineuronal nets in the adult rodent brain. Mol Cell Neurosci 56:186-200.

Welker W, Sanderson KJ, Shambes GM (1984) Patterns of afferent projections to transitional zones in the somatic sensorimotor cerebral cortex of albino rats. Brain Res 292:261-267.

Wu H, Luo J, Yu H, Rattner A, Mo A, Wang Y, Smallwood PM, Erlanger B, Wheelan SJ, Nathans J (2014) Cellular resolution maps of $X$ chromosome inactivation: implications for neural development, function, and disease. Neuron 81:103-119.

Ye Q, Miao Q (2013) Experience-dependent development of perineuronal nets and chondroitin sulfate proteoglycan receptors in mouse visual cortex. Matrix Biol 32:352-363.

Zhou Z, Hong EJ, Cohen S, Zhao WN, Ho HYH, Schmidt L, Chen WG, Lin Y, Savner E, Griffith EC, Hu L, Steen JAJ, Weitz CJ, Greenberg ME (2006) Brain-specific phosphorylation of MeCP2 regulates activity-dependent Bdnf transcription, dendritic growth, and spine maturation. Neuron 52:255-269. 